\title{
WestVirginiaUniversity
}

THE RESEARCH REPOSITORY @ WVU

Graduate Theses, Dissertations, and Problem Reports

2017

\section{Evaluation of Ultrasonic Transducers for Use with Fire Apparatus}

David Cody Lee Cutright

Follow this and additional works at: https://researchrepository.wvu.edu/etd

\section{Recommended Citation}

Cutright, David Cody Lee, "Evaluation of Ultrasonic Transducers for Use with Fire Apparatus" (2017). Graduate Theses, Dissertations, and Problem Reports. 5425.

https://researchrepository.wvu.edu/etd/5425

This Thesis is protected by copyright and/or related rights. It has been brought to you by the The Research Repository @ WVU with permission from the rights-holder(s). You are free to use this Thesis in any way that is permitted by the copyright and related rights legislation that applies to your use. For other uses you must obtain permission from the rights-holder(s) directly, unless additional rights are indicated by a Creative Commons license in the record and/ or on the work itself. This Thesis has been accepted for inclusion in WVU Graduate Theses, Dissertations, and Problem Reports collection by an authorized administrator of The Research Repository @ WVU. For more information, please contact researchrepository@mail.wvu.edu. 


\title{
Evaluation of Ultrasonic Transducers for use with Fire Apparatus
}

\author{
David Cody Lee Cutright \\ Thesis submitted to the \\ College of Engineering and Mineral Resources \\ At \\ West Virginia University \\ In partial fulfillment of the requirements \\ For the degree of \\ Master of Science \\ In \\ Electrical Engineering
}

Roy S. Nutter, Ph.D, LCSEE, Committee Chairperson

Powsiri Klinkhachorn, Ph.D, LCSEE

Vinod Kulathumani, Ph.D, LCSEE

Lane Department of Computer Science and Electrical Engineering Morgantown, West Virginia 2017

Keywords: Ultrasonic transducer, fire apparatus, flowmeter, flow sensor

(C) 2017 David Cutright 


\section{Abstract \\ Evaluation of Ultrasonic Transducers for use with Fire Apparatus}

\section{David Cutright}

Fire departments are tasked with the protection of life and property in dangerous situations. Currently, water consumption for fire response is typically an estimate or a guess by the pump operator.

This thesis evaluates the use and applicability of an ultrasonic Time-of-Flight flowmeter on the discharge of a fire apparatus pump. The tests showed that the Time-of-Flight flowmeter was accurate within ten percent as long as it was at least twenty-five feet from the discharge of the pump. Pump vibration noise seems to prevent accurate use near the pump itself. 


\section{Acknowledgments}

First, a special thank you to my Committee Chair Dr. Nutter, for his direction and guidance over the course of this project. I would also like to thank Dr. Klinkhachorn and

Dr. Kulathumani for serving as committee members and teachers. Additionally, thank you to John Lucas and Jim Hall for their help along the way.

A very large thank you to my family and friends for their support and encouragement over the course of this project.

To everyone who helped or supported me in some form or another on this projectwithout you it would not have been possible.

Incepto ne desistam. 


\section{Table of Contents}

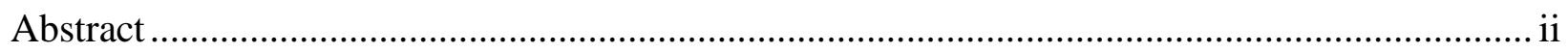

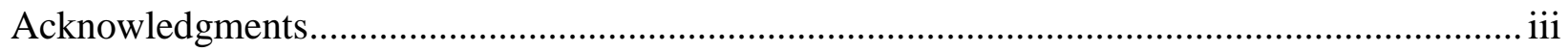

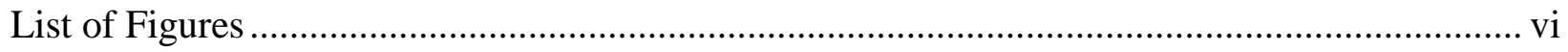

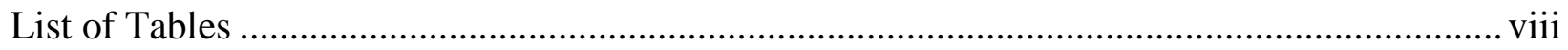

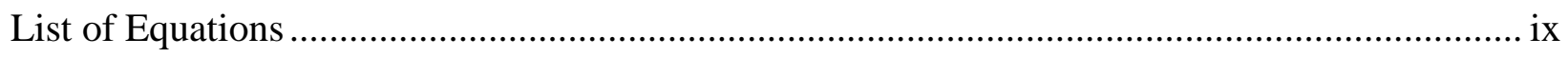

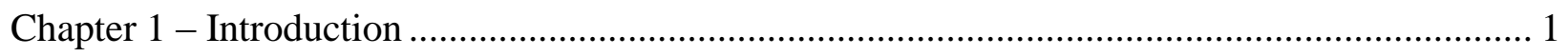

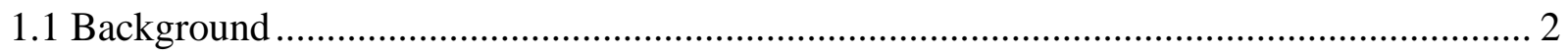

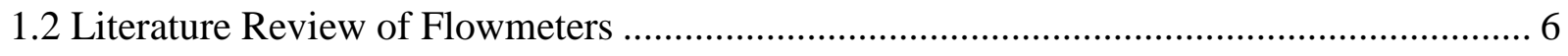

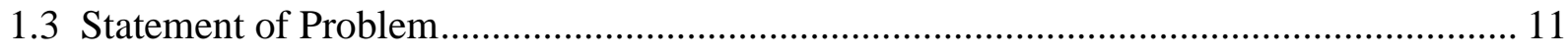

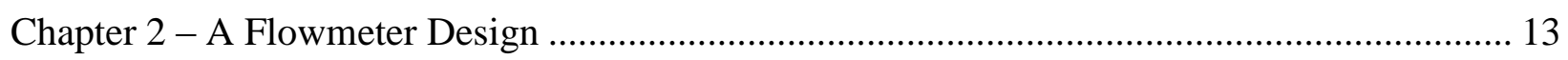

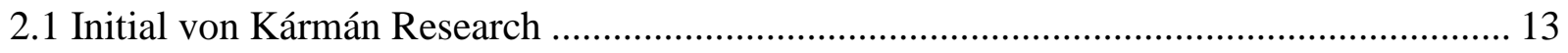

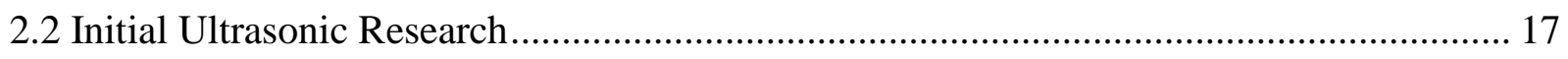

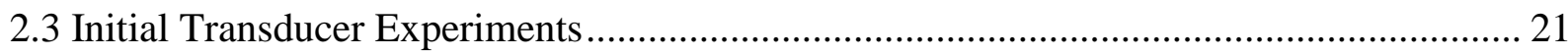

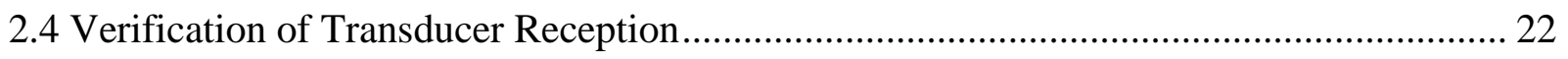

2.5 Sinusoid Transmission with Commercial Product........................................................... 28

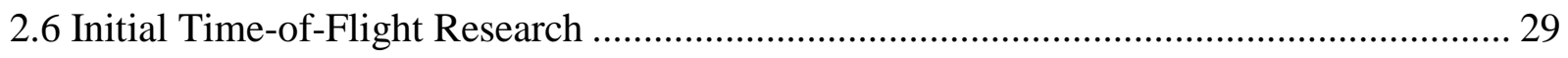

Chapter 3 - Commercial Flowmeter \& Apparatus Experiment ..................................................... 34

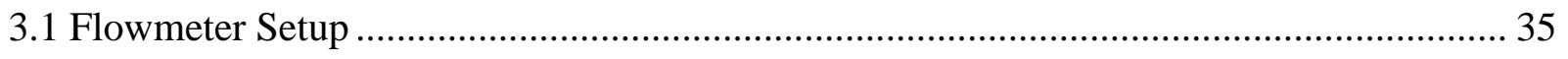

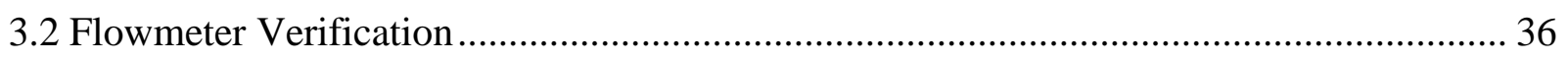


3.3 Flowmeter Variable Parameters

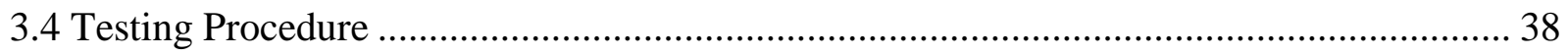

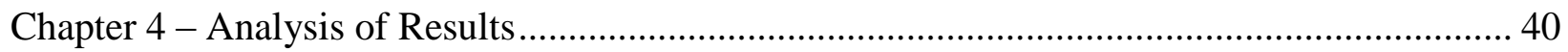

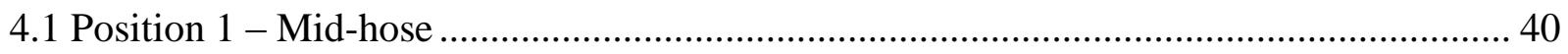

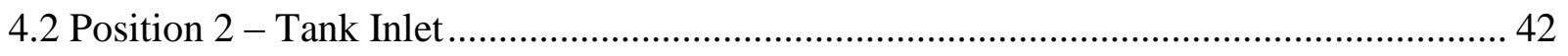

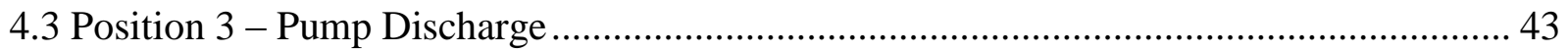

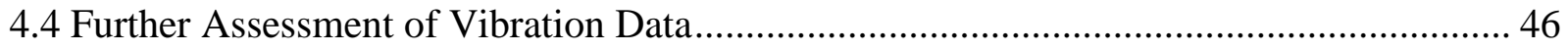

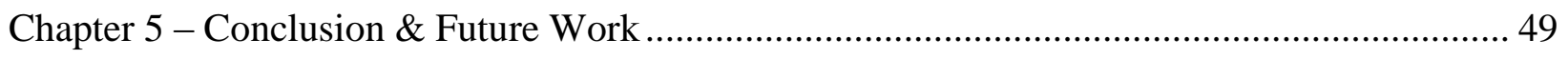

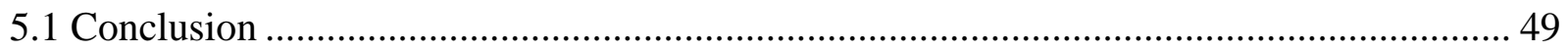

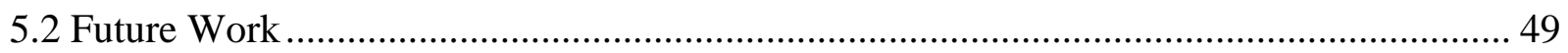

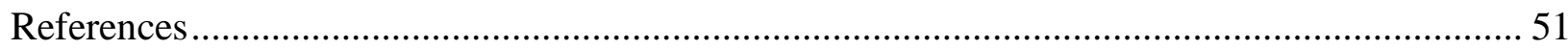

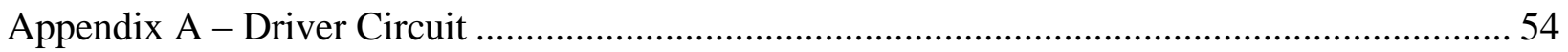




\section{List of Figures}

Figure 1: (A) Branch and (B) Loop Water Distribution [4].................................................... 3

Figure 2: An LED water indicator on a Pierce pumper-tanker apparatus. [6] ................................. 5

Figure 3: Various differential flowmeters [9] .................................................................. 7

Figure 4: a) Paddle wheel style sensor. b) Propeller style sensor. [10] ....................................... 8

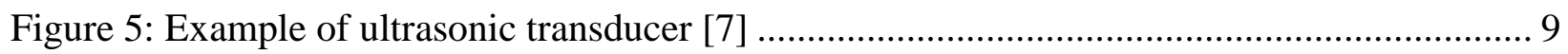

Figure 6: Example of transit-time transducer layout. [7]...................................................... 10

Figure 7: Principle of conventional EMF as illustrated by [13] .............................................. 10

Figure 8: "Visualization of the vortex street behind a circular cylinder in air" [14] .................... 13

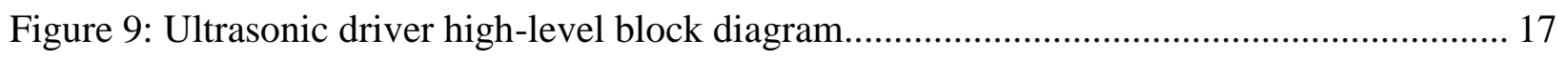

Figure 10: High-level block diagram of devised electrical set-up .............................................. 18

Figure 11: Wiring diagram created in CAD program of two transformers to create isolation ..... 20

Figure 12: Verification of driver circuit output waveform ...................................................... 22

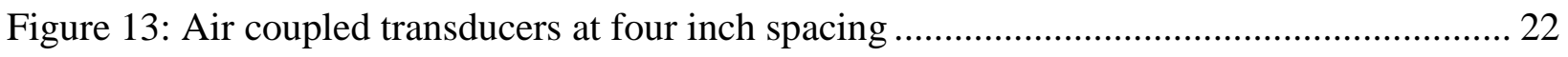

Figure 14: Air coupled transducers at one-half inch spacing ………......................................... 23

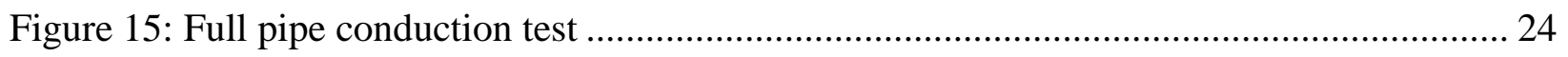

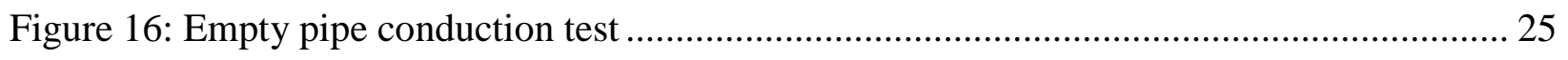

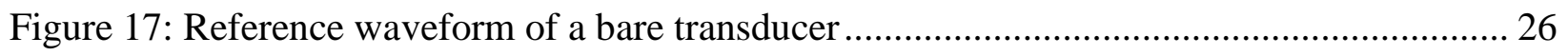

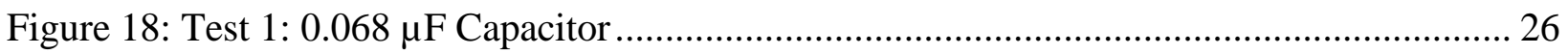

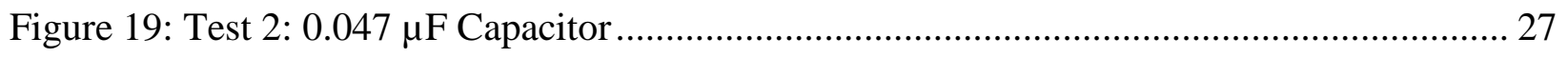

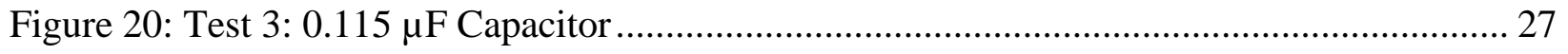

Figure 21: Test 4: $0.115 \mu \mathrm{F}$ Capacitor in Parallel with $10 \mathrm{k} \Omega$ Resistor ......................................... 28

Figure 22: Waveforms with Tektronix AFG2021 at 40KHz ..................................................... 29 
Figure 23: Image of TDC1000-TDC7200EVM [18].

Figure 24: Capture of Texas Instruments Webench Tool Illustrating 45 Degree Transducer

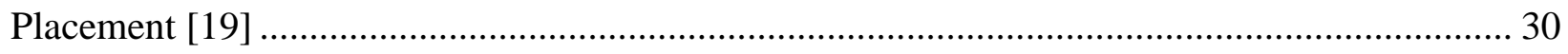

Figure 25: Composite image of a first generation 3-d printed transducer mount...................... 31

Figure 26: Example setup with milled transducer blocks affixed via cyanoacrylate ................. 31

Figure 27: Example oscilloscope output on EVM board ................................................. 32

Figure 28: An example of multiple 'STOP' signals caused by increasing gain ........................ 33

Figure 29: Image of machined adapter hooked to the inlet of a water tender ........................... 34

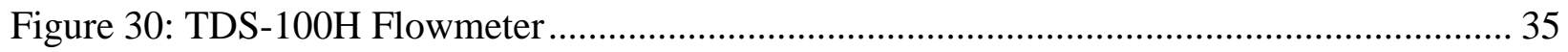

Figure 31: Example of allowable transducer mounting locations [21] .................................. 36

Figure 32: Midline Flowrates at Different PSIs............................................................... 41

Figure 33: Midline Qualities vs Flowrates................................................................... 41

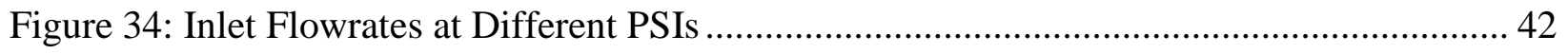

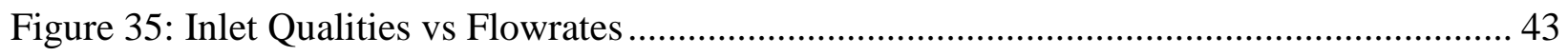

Figure 36: Discharge Flowrates at Different PSIs .......................................................... 44

Figure 37: Discharge Qualities vs Flowrates ................................................................ 44

Figure 38: Flexible Coupling Discharge at Different PSIs ................................................ 45

Figure 39: Flexible Coupling Qualities vs Flowrates ........................................................ 46

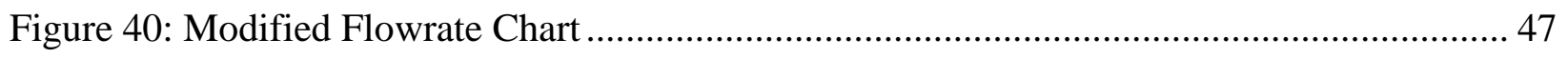

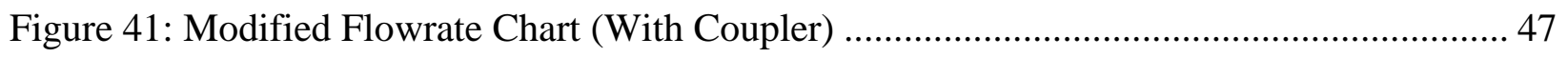

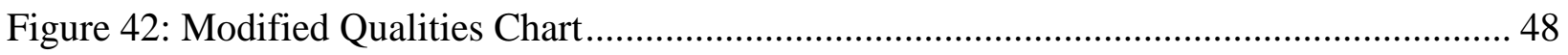

Figure 43: Modified Qualities Chart (With Coupler) ....................................................... 48 


\section{List of Tables}

Table 1: Evaluation of Theoretical Reynolds Numbers............................................................ 16

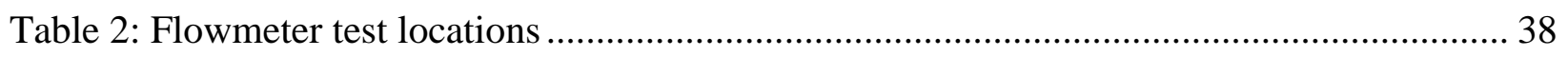

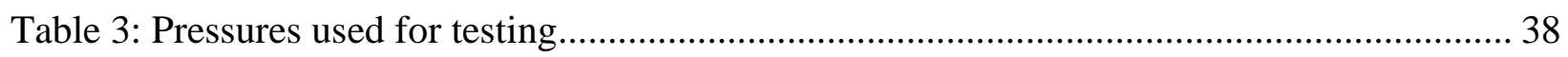

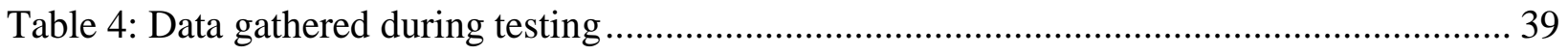




\section{List of Equations}

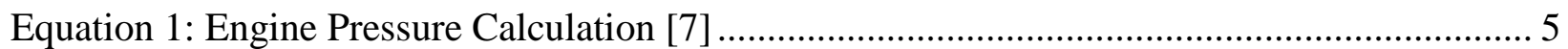

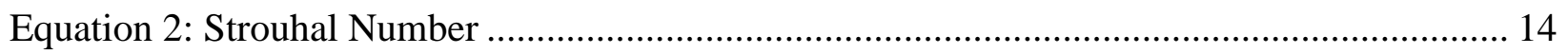

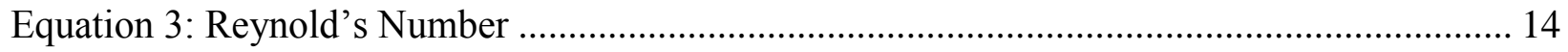

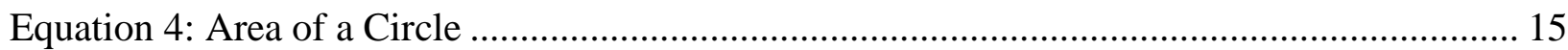

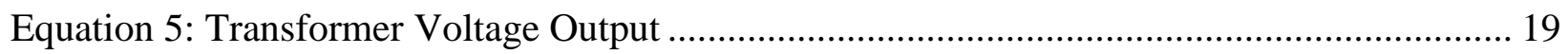

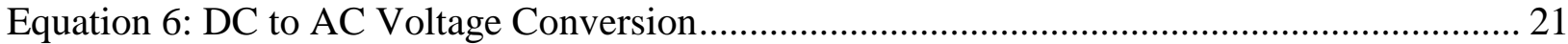




\section{Chapter 1 - Introduction}

The United States fire service is a dynamic industry that strives to adapt new ideas to perform the centuries old mission of fire suppression and mitigation. Some adaptations come in the form of repurposed or developed technologies such as the Self-Contained Breathing Apparatus, which became a requirement for structural firefighting in May of 1971. [1] Other adaptations are modifications to existing strategies such as the reformulation of basic firefighting principles. These adaptations include different water application methods such as ultra-high pressure fog nozzles and the use of ventilation, or lack thereof, to combat dangerous flashover situations. In the context of technology, detailed data about firefighting events is not widely collected due to the nature of firefighting. In 2014, eighty-five percent of fire departments were categorized either as run mainly volunteers or completely by volunteers. [2] Additionally, sixtynine percent of the United States 1,114,750 firefighters were volunteers. The very nature of volunteer firefighting means that the volunteers often work part-time or full-time jobs in addition to their fire service related activities. With this in mind, volunteers may struggle with the time commitment to receive the basic training required to respond to the wide array of calls possible, without imposing additional training on data collection. [3] Therefore, the collection of data is likely to fall to paid fire departments or independent organizations such as the National Fire Protection Agency, U.S. Fire Administration, or Underwriters Laboratories. 


\subsection{Background}

Perhaps the largest universal obstacle of conventional firefighting is the acquisition of a sufficient water source. Without a large enough source of water, fire officials are constrained in their efforts. Fire services secure their water from one of two types of sources: municipal water supplies or static reserves. Each water source presents its own challenges. These challenges are discussed in the following sections.

\subsubsection{Water Sources}

Municipal water supplies are piped systems that normally provide water to residential areas or commercial businesses. These systems are generally reliable but have various limitations. First, municipal systems are only as good as their reservoirs. In periods of drought, the source of the municipality's water can be fragile and fire flow conditions can create an excessive strain. Second, a hydrant must be nearby to make use of such a system. This is typically more of an issue in rural settings where water supplies come from a municipality that is a distance away, and which may not have a large number of hydrants along the main line. The third limitation is flow-rate, often expressed in gallons-per-minute (GPM). Not all water systems are equal. The connection to a single hydrant does not guarantee a sufficient supply of water depending on the scenario. As shown in Figure 1 below, municipal connections either are a branch or loop style layout. A branch setup uses one pipe to supply water to an area and then dead-ends. A loop utilizes two or more pipes to supply an area, which allows for more volume with less overall fluid velocity. 

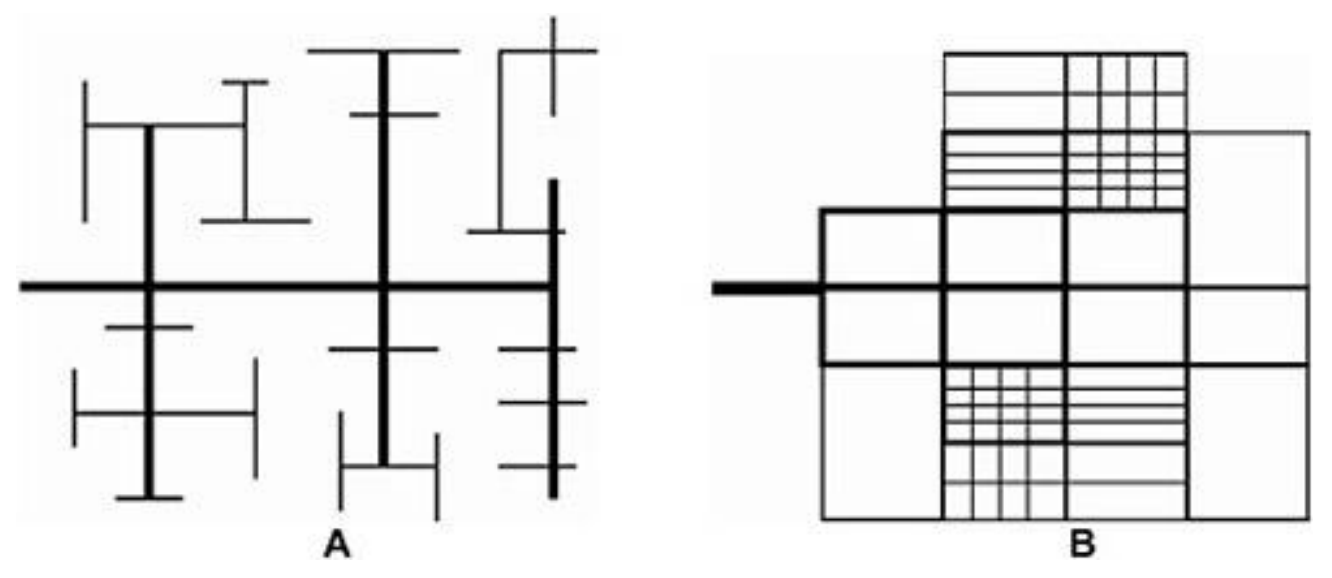

Figure 1: (A) Branch and (B) Loop Water Distribution [4]

The water supply layout is important in areas where attempting to utilize two hydrants on the same branch may outpace the supply capabilities of the pipe and cause a disparity between the desired output and the realized one. It is worth noting that the main requirement for sourcing water from a municipality is flow-rate. The pressure of the water supply is of little consequence, as the fire apparatus will increase the pressure through pumping. In conclusion, municipal supplies may struggle to meet demands associated with a large fire incident, or multiple smaller incidents at the same time.

Static water supplies include ponds, lakes, pools, and streams. These sources often lack piping, although they are occasionally fitted with a dry-hydrant that allows apparatus to more effectively make use of their resources. Static supplies often are poorly accessible compared to their municipal counterparts. Generally, the presence of a large reservoir of water near a fire is purely by chance. A large caveat with static systems is that they require an apparatus to draft from them. Drafting is the process by which a fire apparatus uses a semi-rigid hose and creates a vacuum on the inlet side of its pump. The vacuum causes the water to siphon into the pump, from where it is pumped out to the fire ground. It is worth noting that basic physical limitations restrict the maximum theoretical drafting height to 33.8 feet, although in practice this limit is 
lower. Additionally, per NFPA 1901 any apparatus with a pumper designation is required to carry at least twenty feet of suction hose. [5] Twenty feet may not be of sufficient length to access a static water supply. The use of a static supply essentially ties up one whole apparatus, potentially away from the incident. In conclusion, common issues with static supplies include accessibility and availability.

\subsubsection{Water Delivery}

Regardless of the water source, the water is initially transferred via one of two methods: hose or apparatus. NFPA 1901 requires apparatus with a pumper designation to carry at least 800 feet of $2 \frac{1}{2}$ " or larger hose. [5] If a pumper carrying the minimum amount of hose were more than 800 feet from an incident, multiple apparatus would either have to a) connect in series to reach the incident, or b) connect their supply lines together to allow both (or more) apparatus to be at the incident. This technique is often suitable for urban areas but may not be as practical for rural firefighting. The second option of transferring water is via apparatus. This method typically makes use of fire apparatus designated as water tenders. Water tenders are required to have a capacity of at least 1,000 gallons. [5] The water tenders will fill their tanks at the water source and then drive to the incident, depositing their payload either in another truck or into a portable device known as a dump tank. The water tenders will then begin another circuit to ensure sufficient water supply. Planning and resource management can be beneficial or detrimental to the overall effectiveness of water tender operations.

\subsubsection{Challenges}

The challenges of maintaining a stable supply of water are numerous. Most importantly, a net excess of water must be kept in order to constantly fight fire and ensure the safety of firefighters. It is common for fire apparatus to have general indicators of their reserve water 
levels in the form of LED indicators or incandescent lamps. These indicators show the water level from empty to full. In Figure 2 below, measurements are in increments of twenty-five percent.

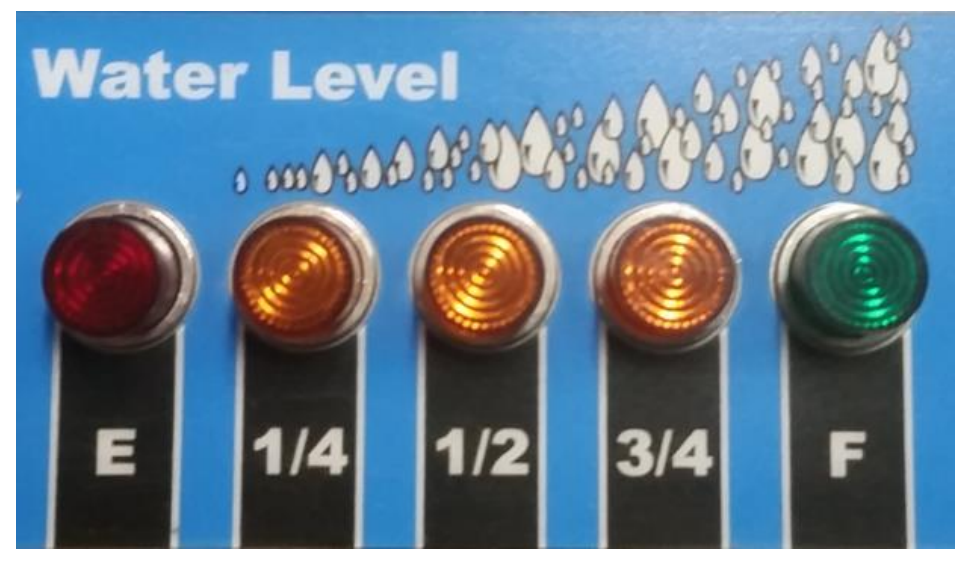

Figure 2: An LED water indicator on a Pierce pumper-tanker apparatus. [6]

In order to keep a general idea of whether or not water demands are being met, pump operators monitor their inlet and outlet pressures and general tank levels, but few if any have the ability to view their gallons-per-minute input and output in real-time. The presence of a pressure reading provides a general idea of the quality of water pressure supplied to firefighters and is often calculated using Equation 1 that follows [7]:

Nozzle Pressure + Friction Loss + Appliance Friction Loss + Elevation Loss or Gain

$$
\begin{gathered}
= \\
\text { Engine Pressure }
\end{gathered}
$$

Determining this number would require lookup tables based on the size, type, and length of hose in use as well as the elevation of the nozzle end. Further complicating this issue, many fire nozzles offer adjustable spray patterns. This means that at any time firefighters have the potential to increase or decrease their water consumption with a change in pressure being the only major indicator to the pump operator. Currently, some fire apparatus monitor select discharge water connections via a small paddle-wheel sensor placed directly in the discharge side 
of their pump. The use and placement of these sensors is apparatus-specific and is by no means standardized.

\subsection{Literature Review of Flowmeters}

In this section, we will review various types of flowmeters and their generally accepted installation criteria, as well as various research on their permissible uses and accuracies.

The general recommendation from flowmeter manufacturers tends to be that a straight section of pipe, at least eight to ten pipe diameters long, be present before a flowmeter. [8] Additionally, general recommendations tend to specify a straight section of at least two pipe diameters be present after the flowmeter. According to Hanson and Schwankl, there is not a clear reason as to why these have become the accepted parameters, as there is not "a clear understanding of [their] origin or the impact on flow measurement devices of upstream flow conditions." [8]

A major requirement of flowmeters tends to be that the pipe be completely full of liquid, with no appreciable air-gap on the topmost side. This ensures that multiple things occur, including the computations based on pipe cross-section are accurate and the meter has sufficient contact with the fluid.

\subsubsection{Differential Meters}

Meters in the differential category typically make use of a narrowing in the piping flowed by a flare out, creating a pressure differential as illustrated below in Figure 3. The differential information is gathered via pressure transducers and used to calculate the flow rate. The transducers therefore must pass through the pipe wall and have contact with the substance being measured to effectively measure the internal pressures. While this is an established principle, it 
was re-articulated as recently as 2014 that these meters, due to the nature of their construction, cause head loss. [9] Head loss is the resistance of flow due to friction, and may or may not be tolerable depending on the application of the meter. For firefighting scenarios, head loss is not tolerable as it impinges on pump efficiency and thereby violates at least one desired characteristic of the outcome of this thesis.

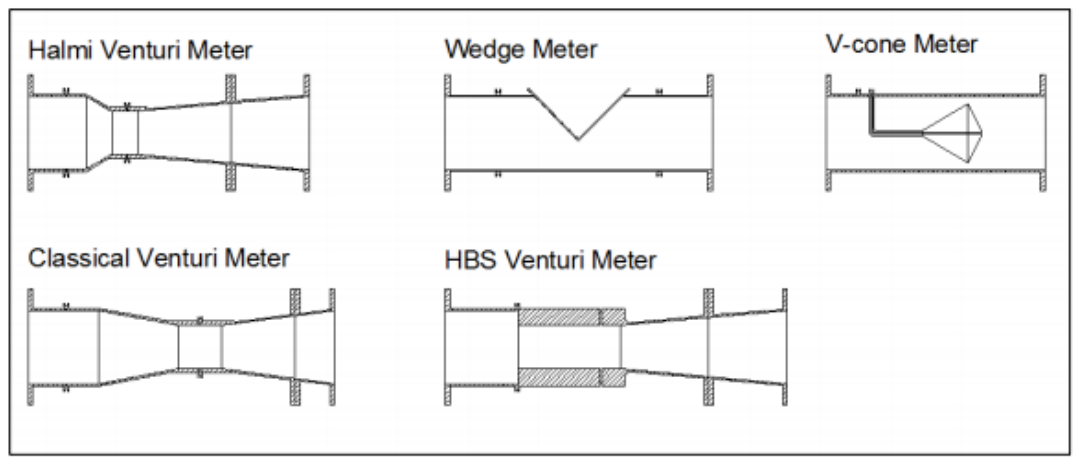

Figure 3: Various differential flowmeters [9]

\subsubsection{Mechanical Meters}

Mechanical meters require physical contact with the sample to generate a reading. The meters are affixed either by use of a saddle clamp or a tee in the piping. This section is not an allinclusive list.

Propeller-style meters make use of a propeller similar to those used for boating. The propeller placement is in the middle of the flow stream oriented perpendicular to the flow, typically via saddle-clamp, and translates the mechanical rotation of the prop to an electrical signal as shown in Figure 4a. Hanson and Schwankl concluded that severe turbulence, as well as propeller diameter, play a large role in error for these meters. [8] Their experiment made use of a partially closed butterfly valve that caused large errors for the propeller meter, regardless of spacing from the valve. 
Paddle-wheel meters are similar to propeller meters in nature. According to apparatus meter websites and e-mail correspondence with United States fire apparatus manufacturers, the paddle-wheel sensor is the predominant sensor currently utilized in fire apparatus. A small paddlewheel is inserted parallel to the flow of the pipe and generates an electrical signal proportional to the flowrate as shown below in Figure 4b. Hanson and Schwankl found in their experiments that paddle-wheel meters tend to perform well when their distance from a disturbance is increased, thus allowing the velocity profile to re-establish. [8]

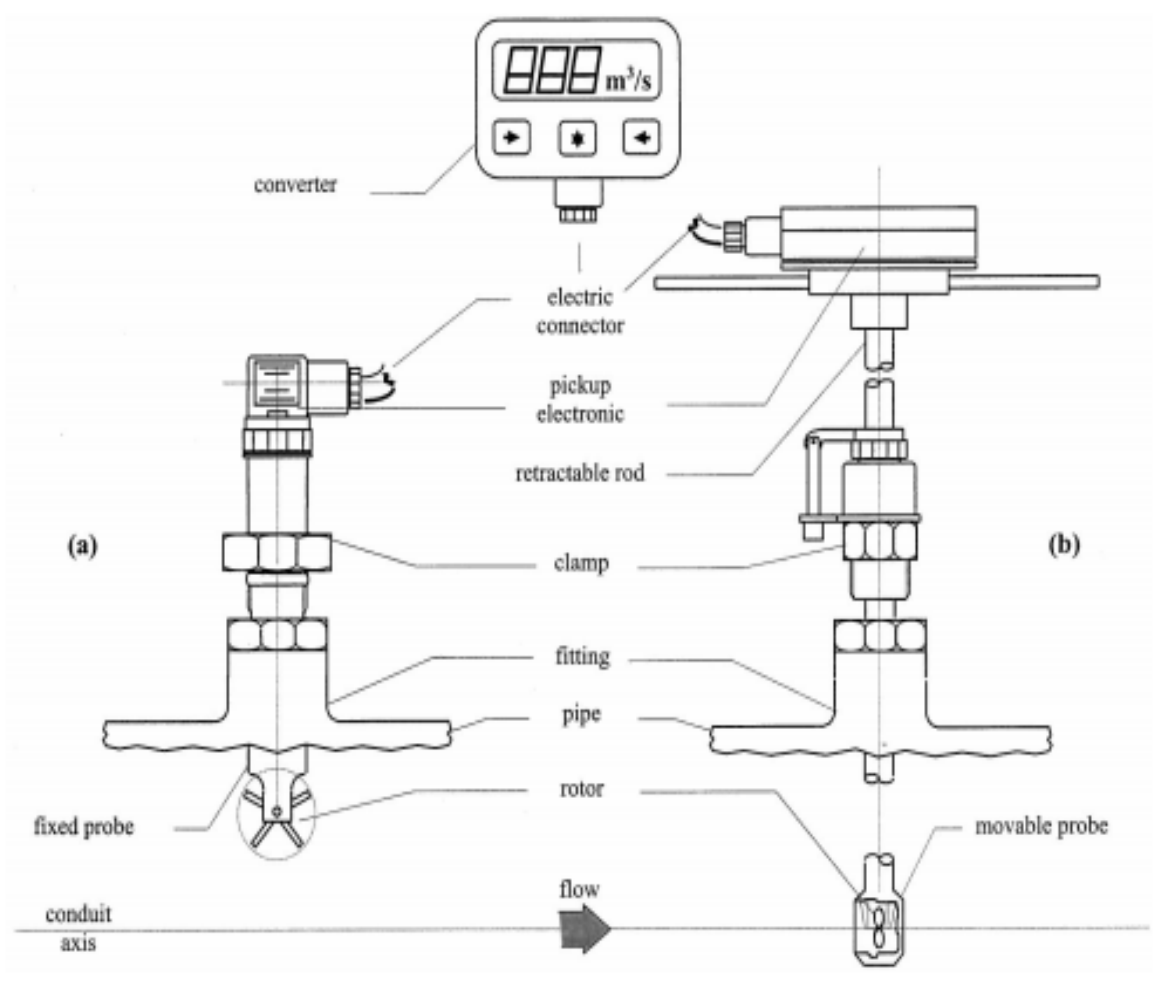

Figure 4: a) Paddle wheel style sensor. b) Propeller style sensor. [10]

\subsubsection{Ultrasonic Flowmeters}

Ultrasonic frequencies are typically above the range of most human hearing and defined as being frequencies in excess of $20 \mathrm{KHz}$ [11]. Ultrasonic flowmeters, also called acoustic 
flowmeters, typically make use of either the Doppler Effect or transit-time measuring at these ultrasonic frequencies to obtain fluid velocity.

The Doppler method works under the assumption that particles moving within a flow travel at the same velocity as the carrier fluid. Additionally, the particles present need to be of sufficient number and size to reflect at least a small portion of the transmitted frequency to the receiver. With this technique, generally two probes are needed - a transmitter and a receiver, although sometimes these probes are co-located within the same housing. Sound waves are generated by the transmitter and pass into a medium at a known frequency; the frequency is reflected by the particles present in the fluid and shifted as a function of the velocity of the fluid as illustrated in Figure 5. The shifted frequency then passes to the receiver. Combining the velocity with the known cross-section of the pipe, a measurement of the total flow can be calculated.

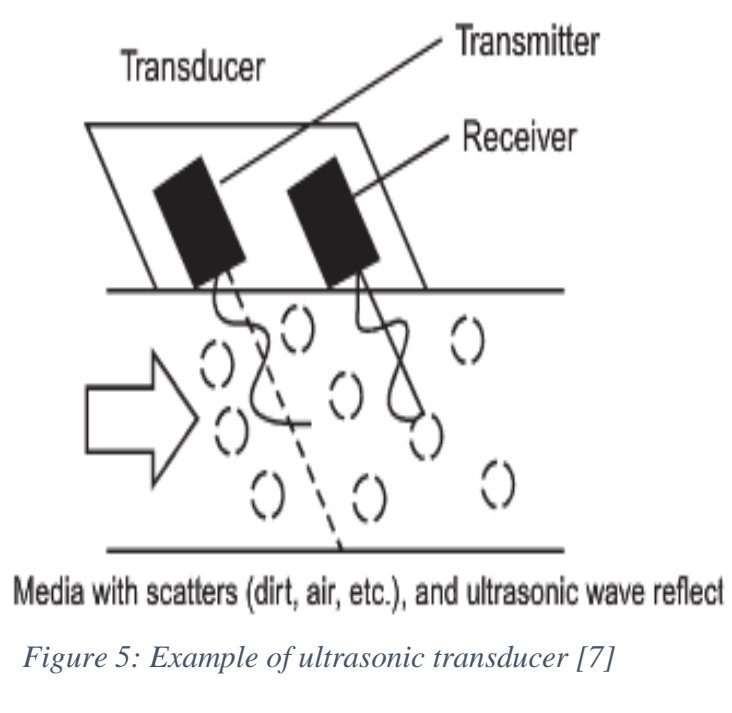

In one study, evaluation of control conditions for the Doppler sensor shows that it typically under-estimated the water flow. [8] The authors believe this may have been attributed to lack of suspended particles within the water. The study went on to show that when coupled with extreme turbulence and a $90^{\circ}$ bend, Doppler sensors do not perform well - regardless of downstream spacing up to ten pipe diameters. 


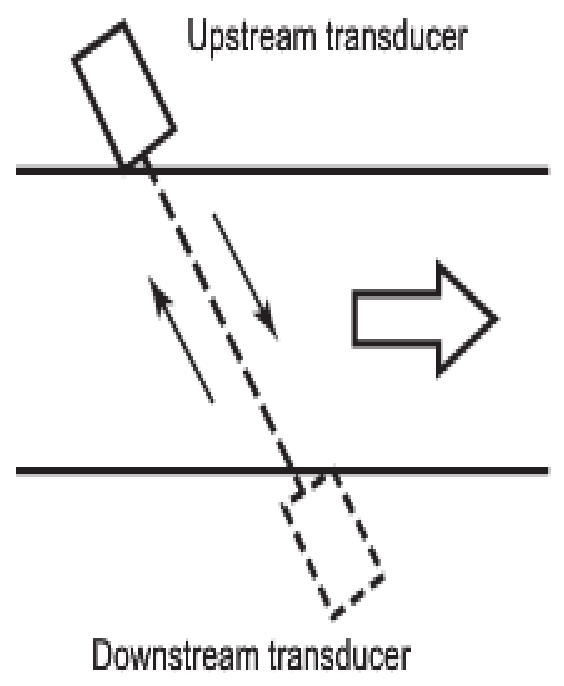

Each transducer alternately acts as a transmitter and receiver.

Figure 6: Example of transit-time transducer layout. [7]
Transit-time meters make use of two probes typically situated on opposite pipe walls as shown in Figure 6 on the left. It is also possible to setup the probes in other methods such as a ' $\mathrm{V}$ ' or ' $\mathrm{W}$ '. In a transit-time meter, two signals are generated rapidly in an alternating fashion: one with the flow of fluid, and one against the flow of fluid. The difference in time it takes the two signals to be received can be used to calculate the velocity of the fluid, and thus with the known cross-section of the pipe the flow rate is calculated. This method is reliable, although turbulence causes some error in measurement. [12]

\subsubsection{Electromagnetic Flowmeter}

Electromagnetic flowmeters are of interest because they have no moving parts. They work by generating a magnetic field across the pipe via two opposing electromagnets. The movement of fluid induces an electrical current between two electrodes

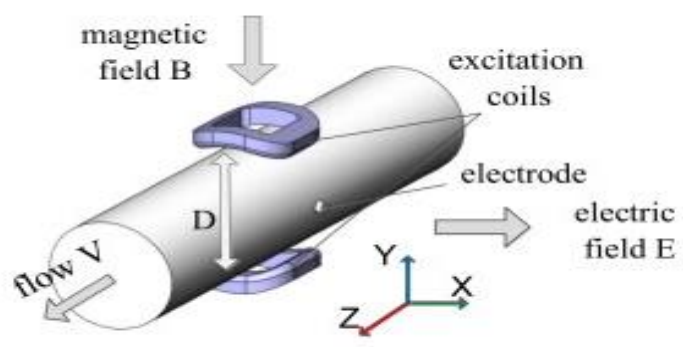
oriented perpendicular to the electromagnets as shown in Figure 7 at the right. With these meters, an uneven flow profile typically causes some fluctuations in readings. 
Work has been conducted by Cao et al. that shows a reduction of measurement errors by optimizing the magnetic field being generated. [13] As a general restriction, for these meters to be effective they require a conductive liquid.

\subsubsection{Vortex Flowmeter}

Vortex flowmeters can be utilized in either open channels or tubing and are of interest because they are used in high Reynolds number applications such as steam measurement Small swirls are created in the medium and measured by a device. The measurements directly correspond to a flowrate. The initial research into this topic stemmed from a discussion of vortex flowmeters and their use in mine air monitoring.

\subsection{Statement of Problem}

It is apparent after reviewing the previous issues that there exists the need for a more comprehensive ability to monitor water usage. The first step in this is to identify if there is a better candidate to monitor flow in firefighting conditions. Inherent problems with developing a more suitable flowmeter for this application include:

- A wide range of flowrates experienced by the apparatus

- Close proximity to causes of turbulence with relation to meter placement

- Varying operating temperatures and environments

- Varying quality of water being pumped

- Multiple intakes and discharges for water

- Usage with both series and parallel fire pumps 
Aside from inherent problems, it is desirable to make improvements to the monitoring devices with regards to accurate measurements and reduced maintenance. Newly applied meters will ideally have the following characteristics:

- Be accurate for a large range of Reynolds numbers

- Consist of few, but ideally no, moving parts

- Be relatively safe from possibly damaging debris in the water supply

- Refrain from impinging on pump efficiency and capacity

- Possess the ability to deliver stable results over the life of the meter

- Lack the requirement of mechanical maintenance

- Be easy to replace if the need arises

It is therefore the objective of this thesis to attempt to build and analyze the suitability of ultrasonic flow sensors to fire apparatus. Specifically, the use of ultrasonic flow sensors that utilize Time-of-Flight calculations at approximately one foot from the discharge of a fire apparatus.

The realization of this goal with the previously mentioned desirable characteristics would allow fire officials to track and analyze their water consumption with little to no additional effort. With this in mind, such information could be passed on to local water providers as well as to organizations such as the National Fire Protection Association and Underwriters Laboratories for calculating statistics on the amount of water required to extinguish fires in various types of building construction. This information can assist with pre-planning how to combat fires in existing structures as well as potentially identifying construction techniques or materials that would require less water to extinguish. 


\section{Chapter 2 - A Flowmeter Design}

This chapter covers early theories, research, and designs explored for use with a flowmeter.

\subsection{Initial von Kármán Research}

Initially, the goal was to create a flow measurement device based on a von Kármán Vortex Street. Theodore von Kármán observed the creation of swirling vortices in fluids once they passed an object. The area where this phenomenon occurs is the 'Kármán Vortex Street' in his honor. A von Kármán vortex street can occur when fluid flows past a bluff body. Under the right conditions, as the fluid flows past the body it will generate alternating vortices as seen below in Figure 8.

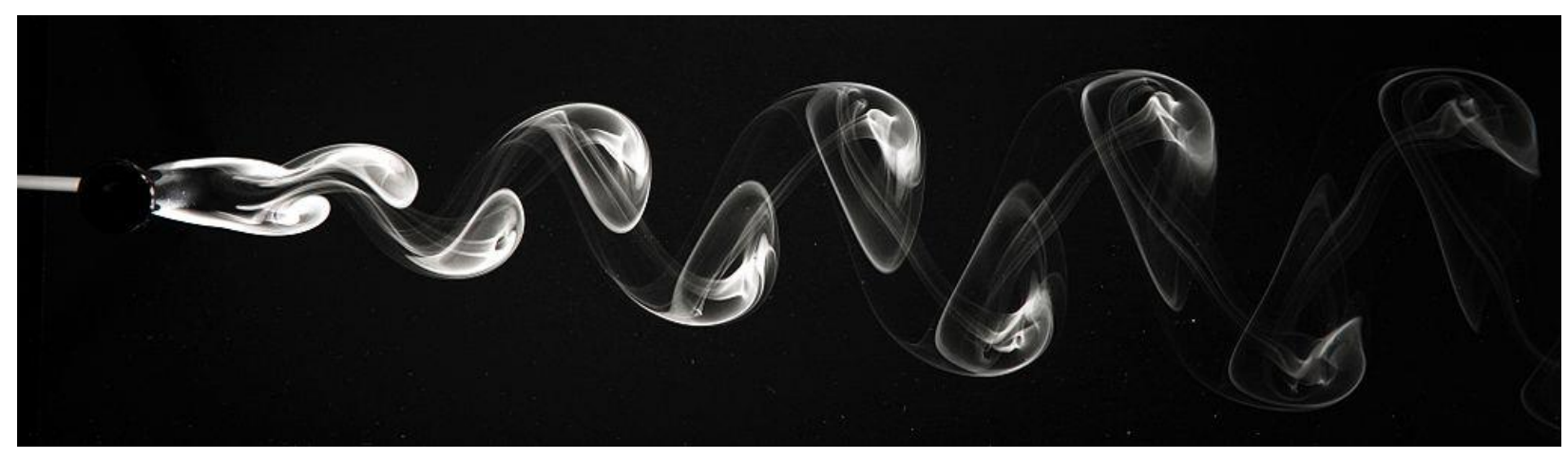

Figure 8: "Visualization of the vortex street behind a circular cylinder in air" [14]

The theory was to insert a small cylindrical body inside of a pipe and attempt to measure the vortices produced by using either light or sound waves. Light was ruled out as the application to fire apparatus does not guarantee a clean water source relatively free of debris. Without a clean water source, the method is unreliable at best as the light would need to be constantly calibrated due to changes in the opacity of the water. We selected sound waves with the theory being that their interaction with vortices would alter their frequency. Any shift in frequency 
would occur from interaction with vortices, thus delivering an accurate vortex count. With this count, we would make use of the Strouhal number to derive the velocity using the equation:

$$
S t=\frac{\omega l}{V}
$$

where $S t$ is the Strouhal number, $\omega$ is the frequency of vortex oscillation, $l$ is the characteristic length, and $V$ is the velocity.

However, it became clear that this approach would be difficult to implement as turbulent flow could possibly mask the vortices and require a very in-depth knowledge of signal processing. We were able to verify that laminar flow would not exist by examining the Reynold's number of the fluid. The Reynold's number is named after British engineer Osborne Reynolds "who first demonstrated that this [...] could be used as a criterion to distinguish between laminar and turbulent flow." [15] To find the Reynold's number, use Equation 3

$$
R e=\frac{\rho V l}{\mu}
$$

where $R e$ is the Reynold's number, $\rho$ is the density of the fluid, expressed in $\mathrm{kg} / \mathrm{m}^{3}$ or $l b m / f t^{3}$, $V$ is the velocity based on the cross section of the pipe, $l$ is the characteristic length, and $\mu$ is the viscosity of the fluid expressed in $N * s / \mathrm{m}^{2}$ or $\mathrm{kg} / \mathrm{m} * s$. The fluid flow in a round pipe is laminar if the Reynolds number is less than approximately 2,300, and is turbulent if greater than approximately 4,000. [15] [16] In between these two numbers the flow may transition between turbulent and laminar, this is transitional flow. 
To examine the Reynold's number, we assumed a flow rate of 250 gallons-per-minute in a 5" diameter pipe. The area of the cross section of the pipe is using

$$
A=\pi r^{2}
$$

for a result of $19.6349 \mathrm{in}^{2}$. One cubic foot contains approximately 7.4805 US gallons of water, with each gallon occupying roughly $231 \mathrm{in}^{3}$. Based on the previous two numbers, a gallon of water occupies approximately 11.7647 inches of 5-inch diameter pipe. With 4 1/6 gallons moving past a point each second and each gallon occupying 11.7647 inches, the fluid flows at approximately $49.01879902 \mathrm{in} / \mathrm{s}$ or $1.245 \mathrm{~m} / \mathrm{s}$. Assuming a water temperature of 75 degrees Fahrenheit gives a density of approximately $997.3268737 \mathrm{~kg} / \mathrm{m}^{3}$. With these variables, we can solve the Reynolds Formula and see that the Reynolds number is just over 177,000 - well past the boundary of 4,000 for turbulent flow in a pipe. 
Further calculation of the Reynolds number for varying pipes and fluid flows yielded the following table ${ }^{1}$ :

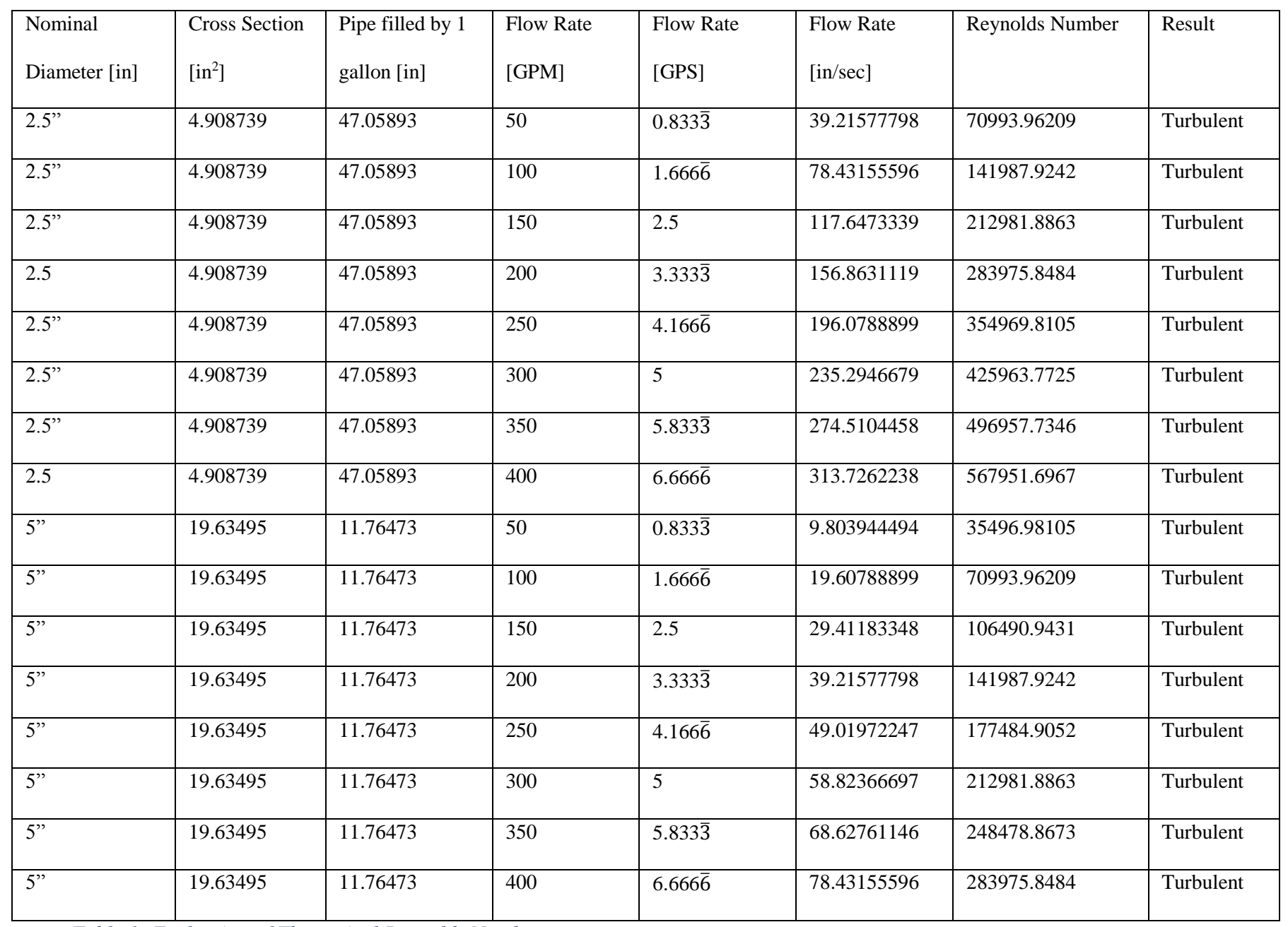

Table 1: Evaluation of Theoretical Reynolds Numbers

During additional literature review of these flowmeters, the need for routine maintenance was apparent. It was unclear if the bluff body itself required cleaning or maintenance was required of the small 'toggle' that senses vortices in the mechanical versions. Additionally, some meters including 'straightening' vanes to reduce turbulent flow. The use of vanes pointed toward a potentially undesirable outcome in violation of our desired goals by placing objects in the pipe

\footnotetext{
${ }^{1}$ All temperatures were assumed to be 75 degrees Fahrenheit density of approximately $997.3268737 \mathrm{~kg} / \mathrm{m}^{3}$.
} 
in addition to the required bluff body - it was unclear how the vanes could affect ultrasonic measurements attempting to count vortices. With this insight now in hand, research turned to an application of ultrasonics that would not require the use of vortices.

\subsection{Initial Ultrasonic Research}

For ultrasonics, the first hurdle was the creation of a device that could generate the frequencies needed to drive the ultrasonic transducers. This device is necessary for a transit-time approach or a Doppler Effect approach.

\subsubsection{Driver Circuit}

The driver circuit appears in Appendix A. The selected driver circuit is a modified version of an ultrasonic driver designed by Lindsay Wilson - with permission. [17] Several modifications are made to the driver circuit. The first modification was to replace the voltagecontrolled-oscillator (VCO) with an Arduino Uno R3. The next modification was the removal of the built-in voltage and current meter. In its entirety, the driver circuit high-level block diagram consists of a frequency generator (Arduino), digital control logic, and analog output circuitry shown below in Figure 9.

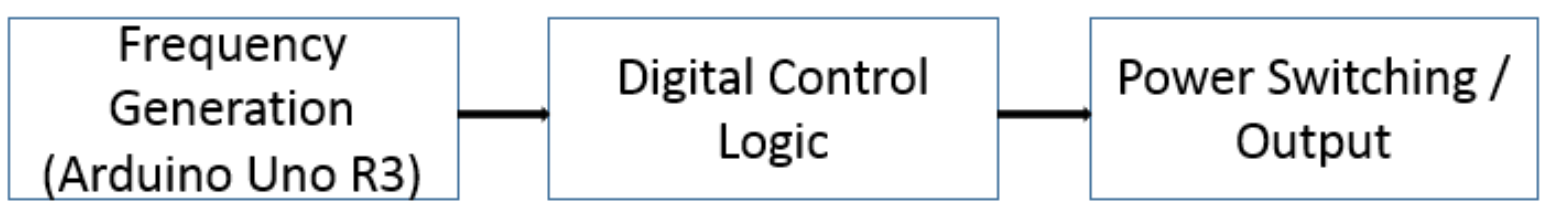

Figure 9: Ultrasonic driver high-level block diagram

\subsubsection{Digital Logic Circuitry}

The Arduino's output was fed into digital logic which ultimately controlled the output of power from the circuit. NOR gates onboard a 7402 integrated circuit is used to generate a pair of opposing logic signals at the same time, which then run to an IR2110 integrated circuit. The 
IR2110 is a high and low side driver that serves the purpose of amplifying the voltage of the digital logic to a level that is suitable for MOSFET control. Essentially, the IR2110 acts as a protective buffer between the low voltage of the digital logic from the relatively high voltage of the output.

\subsubsection{Analog Output Circuitry}

Figure 10 below shows the analog output circuitry. The output from the digital logic is fed directly into the gates of two STP13NK60Z N-channel MOSFETs. More specifically, pins 1 and 7 of the IR2210 were each connected to pin 2 of one of the MOSFETs. The MOSFETs, when activated, allowed the DC current to be switched on and off at the desired frequency.

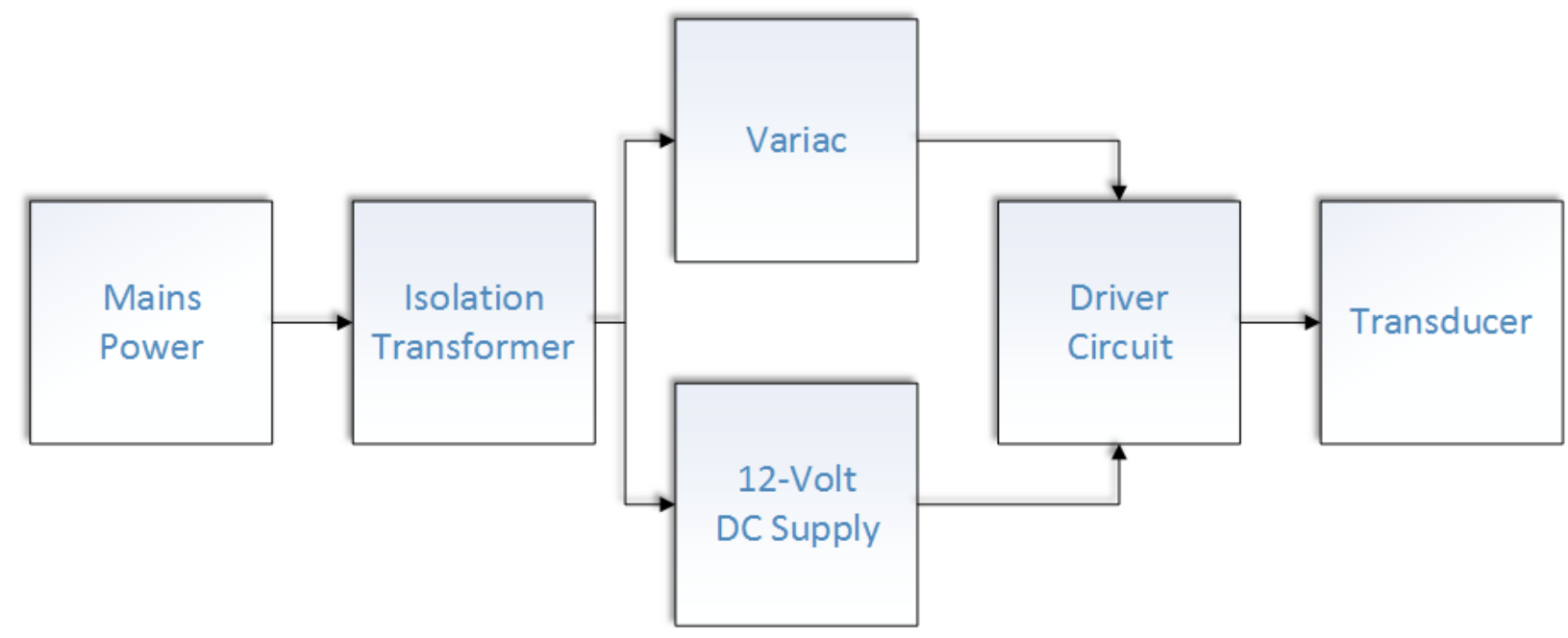

Figure 10: High-level block diagram of devised electrical set-up

With the analog circuitry complete, attention turned to the production of the voltages required to power the MOSFETs.

\subsubsection{Isolation Transformer}

The purpose of an isolation transformer is to provide an overall increase of safety while working with a Device Under Test (DUT). In general, the transformer achieves this by leaving 
the secondary, or output, stage ungrounded. This causes the output voltage to no longer reference the Earth as ground. At this point, if a DUT were to have an unknown short circuit while a user was touching its housing they would be in relative safety - as the voltage would attempt to conduct back to the output of the transformer, instead of through the user to the ground. Typically, isolation transformers have an equal amount of primary and secondary windings, therefore creating a 1:1 voltage ratio in accordance with the following Equation 5:

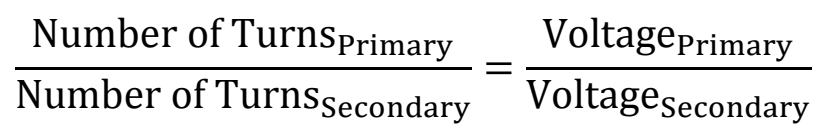

Isolation transformers are useful on devices that require oscilloscope testing. It is important to note that isolation transformers are for use on the DUTs, and not the oscilloscopes themselves. An oscilloscope's chassis is typically connected to Earth ground. Without an isolation transformer on the DUT, it would only require a minor tremor in a user's hand to effectively short non-isolated AC current through the oscilloscope's case. If the isolation transformer were to be connected to the oscilloscope instead of the DUT, the oscilloscope would effectively "float" at whatever dangerous voltage it met. The result of this scenario is that the user would potentially complete a short circuit (read electrocuted) through the oscilloscope to Earth ground.

A 1:1 isolation transformer was not available for use. The experiment set-up instead utilized two transformers to create an isolation transformer as shown in Figure 11 below. The primary windings of the transformers are $120 \mathrm{VAC}$ at $300 \mathrm{~mA}$ and the secondary windings are 12.6VAC at $3 \mathrm{~A}$. The secondary windings were connected together to step 120VAC down to 12.6VAC, and then back up to $120 \mathrm{VAC}$ - effectively creating an isolation transformer with a 
one-to-one ratio. The center taps are disconnected to minimize the chance of imbalance in the 12.6V secondary windings.

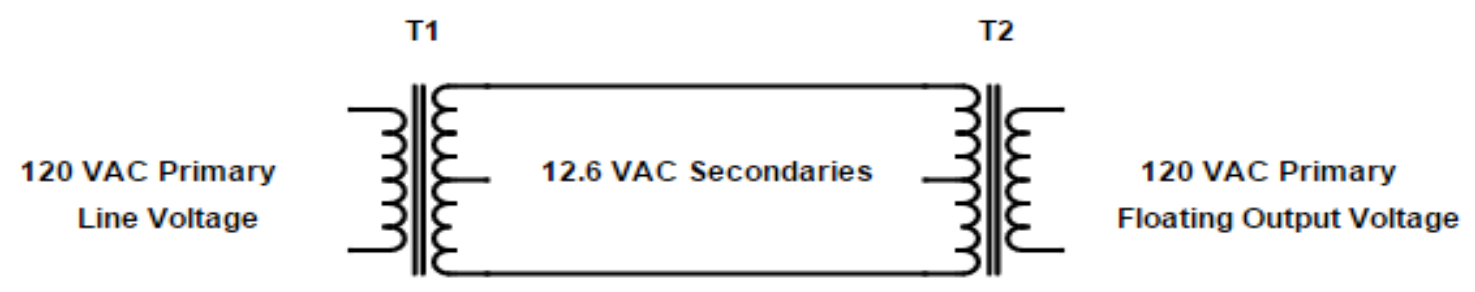

Figure 11: Wiring diagram created in CAD program of two transformers to create isolation

The transformers are in relatively close proximity within their housing. In an attempt to reduce interference between the core magnetic fields, the transformers are at an approximate right angle with respect to each other.

\subsubsection{Variable Transformer}

The function of variable transformers (often generically referred to by the brand name Variac) is to adjust main-line voltages from an outlet. A key feature of these devices is that they have one winding. As such, they provide no isolation between their power source and any connected devices. As their name implies, the output voltage can be varied. Generally, this happens via a dial. In a typical construction, there is a sliding brush which moves over the winding. This brush is connected to the input $\mathrm{AC}$ voltage while the other end of the winding is connected to the outlet, and consequently the powered device. Moving the brush back and forth allows the user to change the number of wires actively being used by the transformer in accordance to Equation 5, thereby changing the output voltage. While there are many different ranges of capabilities, most of the ones within the price range of this project produced anywhere from 0 to 130 volts on the output. 
A variable transformer is desirable because different ultrasonic transducers allow different maximum voltages peak-to-peak (Vpp). For the purpose of this experiment voltages were kept at 10VDC, yielding 20Vpp when converted to a switched output. This was the maximum Vpp of the oscilloscope in use. To achieve 50VDC, approximately 78.616VAC is required in accordance with the following Equation 6:

$$
\mathrm{AC}=\frac{\mathrm{DC}}{0.636}
$$

\subsection{Initial Transducer Experiments}

Initial verification of waveforms uses a PicoScope 2204A USB oscilloscope. Advantages of this device over traditional oscilloscopes include the ability to apply filters and label the various peaks and troughs. Additionally, the use of a USB oscilloscope lends itself to highquality images of the waveforms. A noted drawback of this particular model of PicoScope is a maximum peak-to-peak voltage of 20 volts.

\subsubsection{Verification of Ultrasonic Driving Circuit Output}

The first experiment served to verify that the driver circuit was in fact outputting a square wave. The experiment placed a resistor across the output wires of the MOSFETs and attaching the PicoScope on both sides. This experiment also creates a baseline for the frequency at which the generator was operating.

The results in Figure 12 below show a duty cycle of approximately $49.6 \%$, a peak-topeak voltage of approximately 17.19 Volts, and a frequency centered on 39.96 Kilohertz. These settings are in use for the following experiments. 


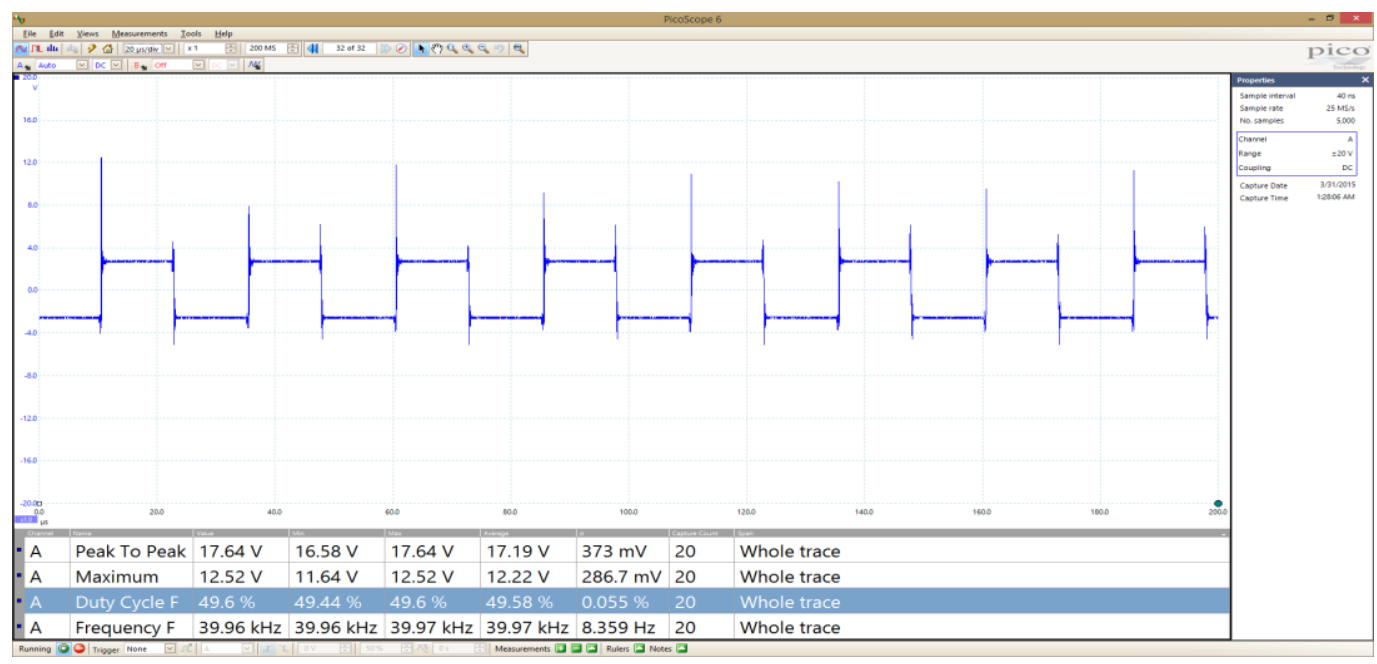

Figure 12: Verification of driver circuit output waveform

\subsection{Verification of Transducer Reception}

The purpose of these experiments is to verify that the signal sent by one transducer is correctly received by the second.

\subsubsection{Four inch spacing with air-coupling}

The transducers were air-coupled at a distance of four inches. In Figure 13 below, the blue line is the signal generated by the emitting transducer. The red line is the received signal.

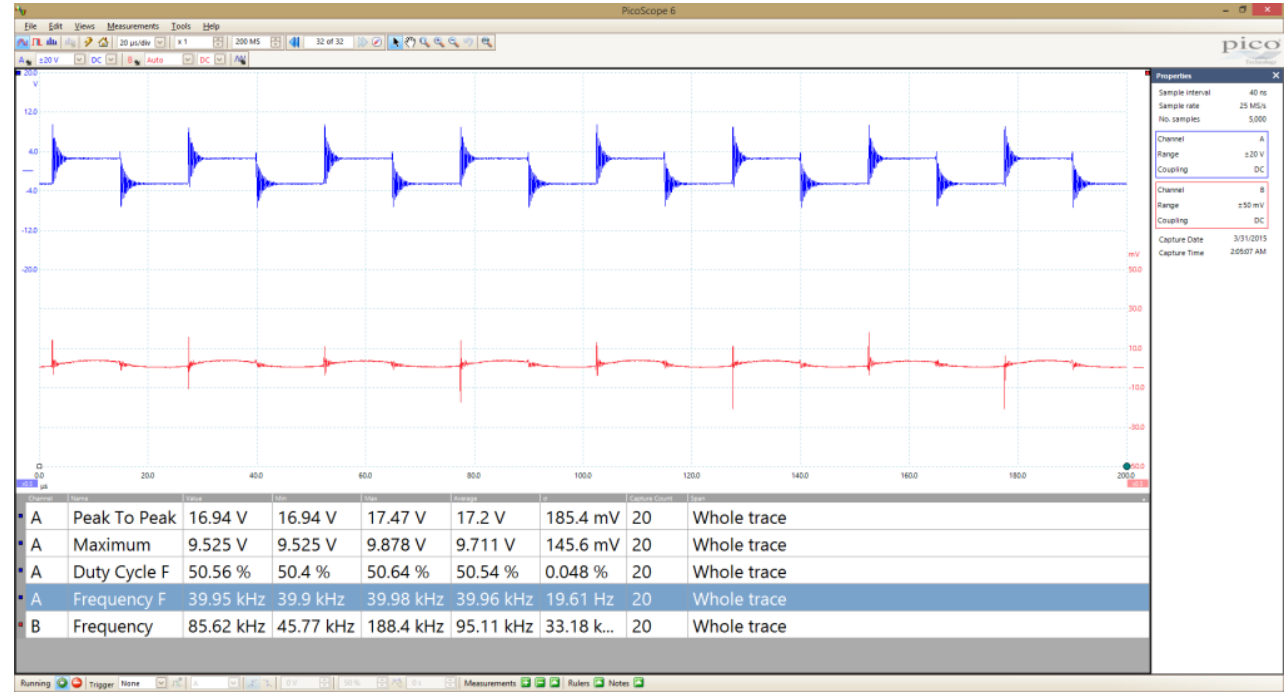

Figure 13: Air coupled transducers at four inch spacing 
The first obvious difference from the baseline experiment is the ringing induced on the leading edge of the transmitter. A second observation was that the received waveform appeared to be a sinusoid and not a square waveform. Although obscured in this image, the received waveform was on the order of millivolts.

\subsubsection{One-half inch spacing with air-coupling}

In the Figure 14, the same test in 6.2 .1 was ran, but this time with a spacing of $1 / 2 "$.

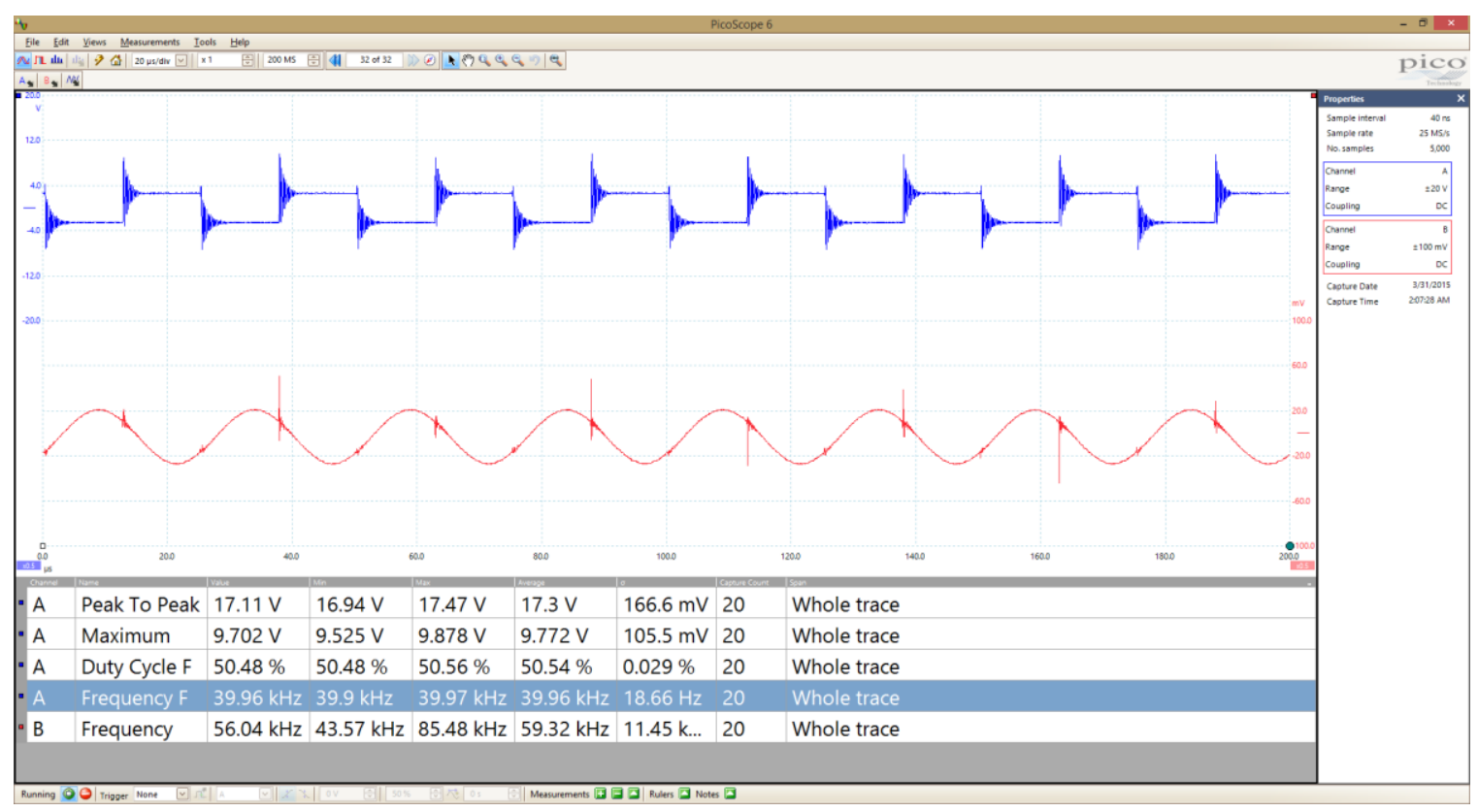

Figure 14: Air coupled transducers at one-half inch spacing

This set-up showed a distinct sinusoid forming that also contained the ringing effect shown in the transmitter. Interestingly enough the ringing received by the second transducer presents itself more clearly during the change from negative to positive voltage on the transmitter. Only minor ringing is received when the transmitter changes from positive to negative voltage. 


\subsubsection{Pipe testing}

At this point, it was verified that a transmitted signal could be discerned by a receiver via air. This next experiment served to test whether or not a pipe wall would successfully transmit the signal between the two transducers.

In this setup, both transducers are attached to the same side of a 3" PVC pipe that had a flat surface milled onto it. Ultrasonic coupling gel is present to ensure transmission of the waves into the pipe wall. The transducers were spaced 4" on center.

The first test results shown in Figure 15, utilizes the setup described above with the only change being the pipe is completely filled with water to remove any air.

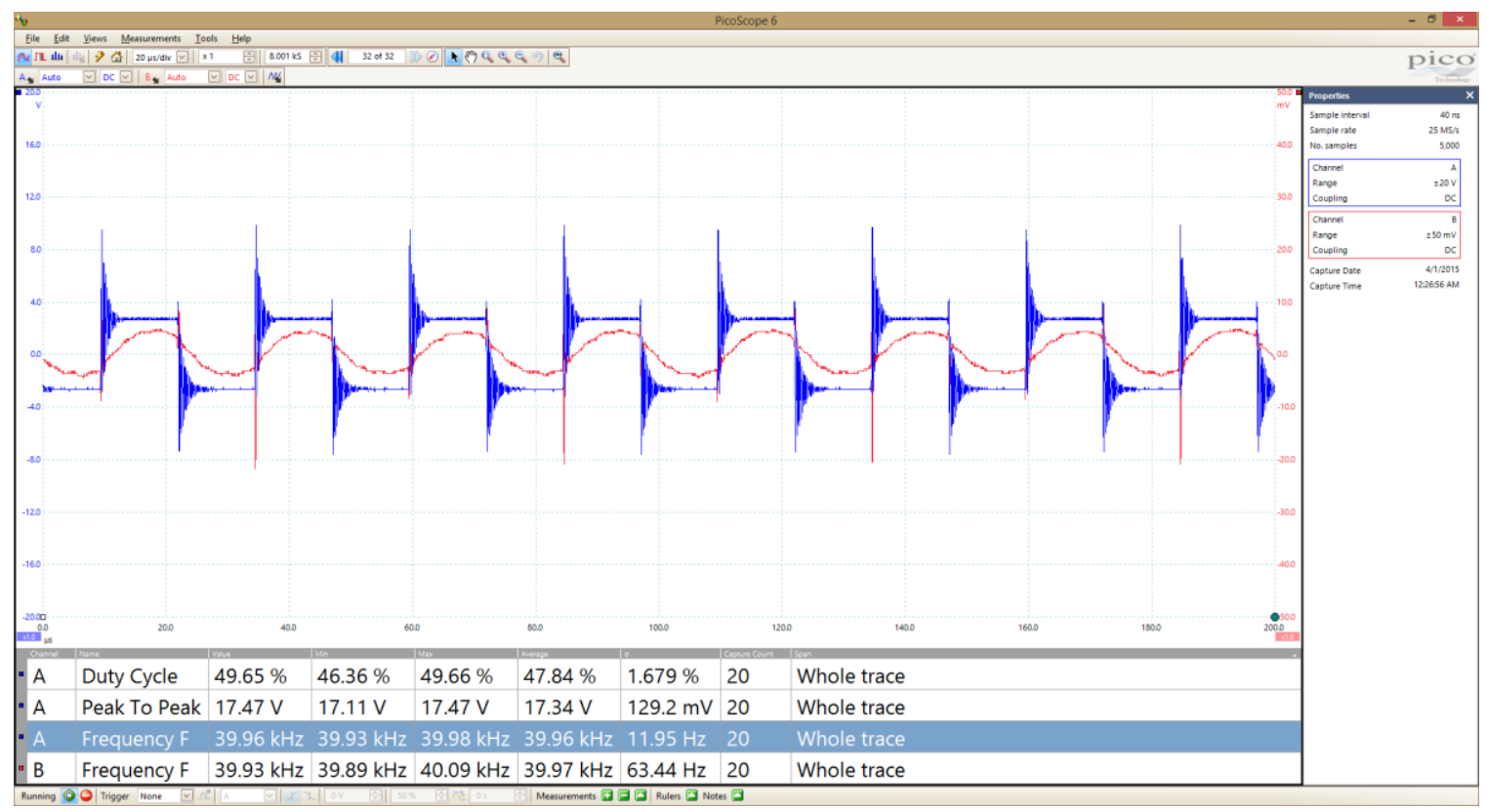

Figure 15: Full pipe conduction test

This seemed to indicate signal transmission through the water, but was not definitive proof. Note that the blue line is on a scale of \pm 20 Volts along the entire axis and the red line is on a scale of \pm 50 millivolts across the entire axis. 
In Figure 16 below, the same setup as above is in use, however this time the pipe is empty. The same fittings and caps are in place as though it is full of water.

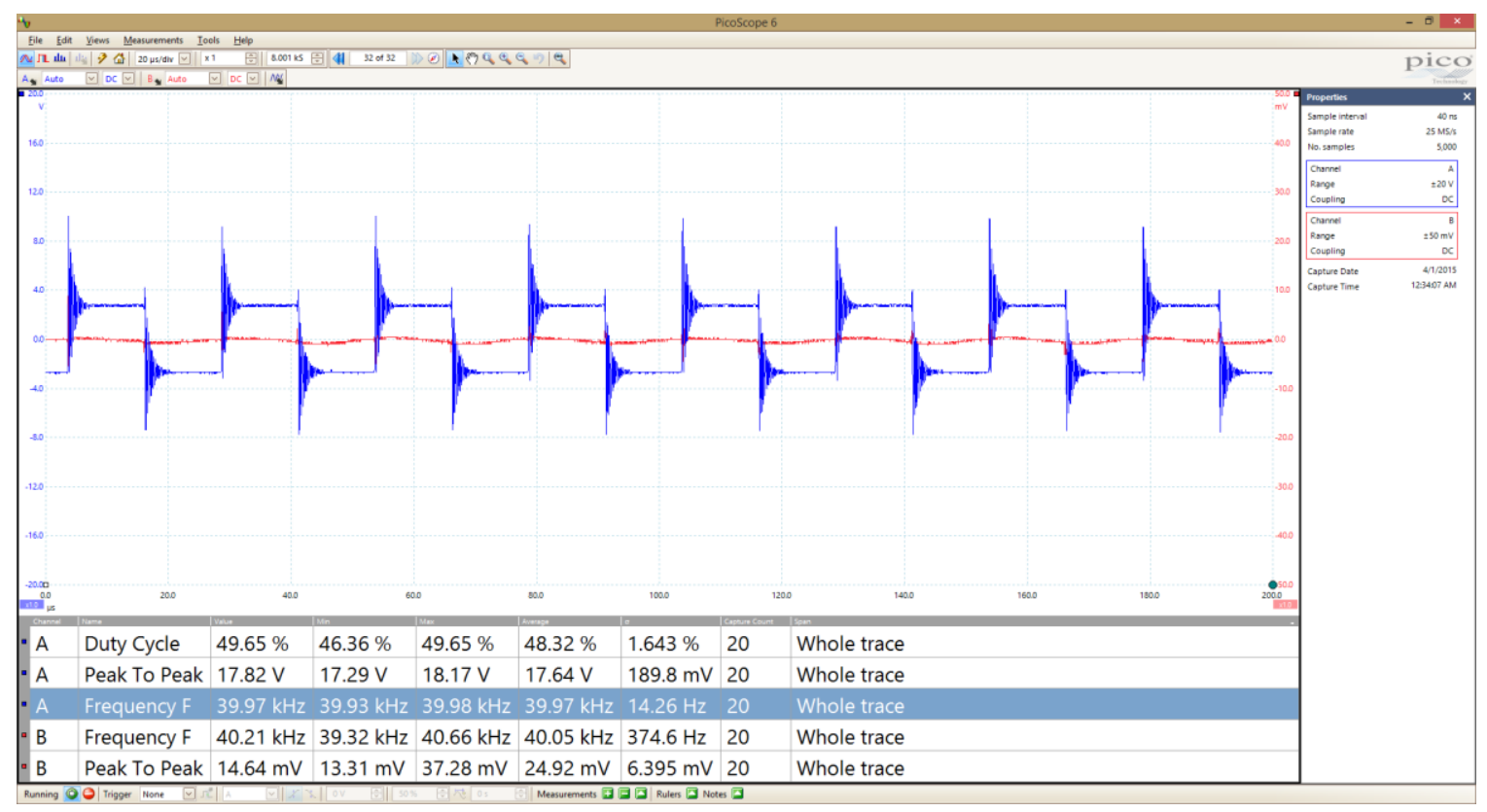

Figure 16: Empty pipe conduction test

While the PicoScope shows approximately $40 \mathrm{KHz}$ on the received frequency, there is a definitive lack of amplitude of the waveform. The transducer's placement is correct and found to be resting flat against the milled pipe surface as in previous tests.

\subsubsection{Investigation of Ringing}

A major concern was that the ringing of the transmitting transducer would cause unnecessary interference in the medium and obscure results. To help reduce this, tests were performed using non-polarized capacitors to attempt to either produce a cleaner square wave or convert the wave into a clean sinusoid. The results are shown below in Figures 17 through 21. In an effort to obtain a cleaner waveform the voltage was approximately 12 volts peak-to-peak. 

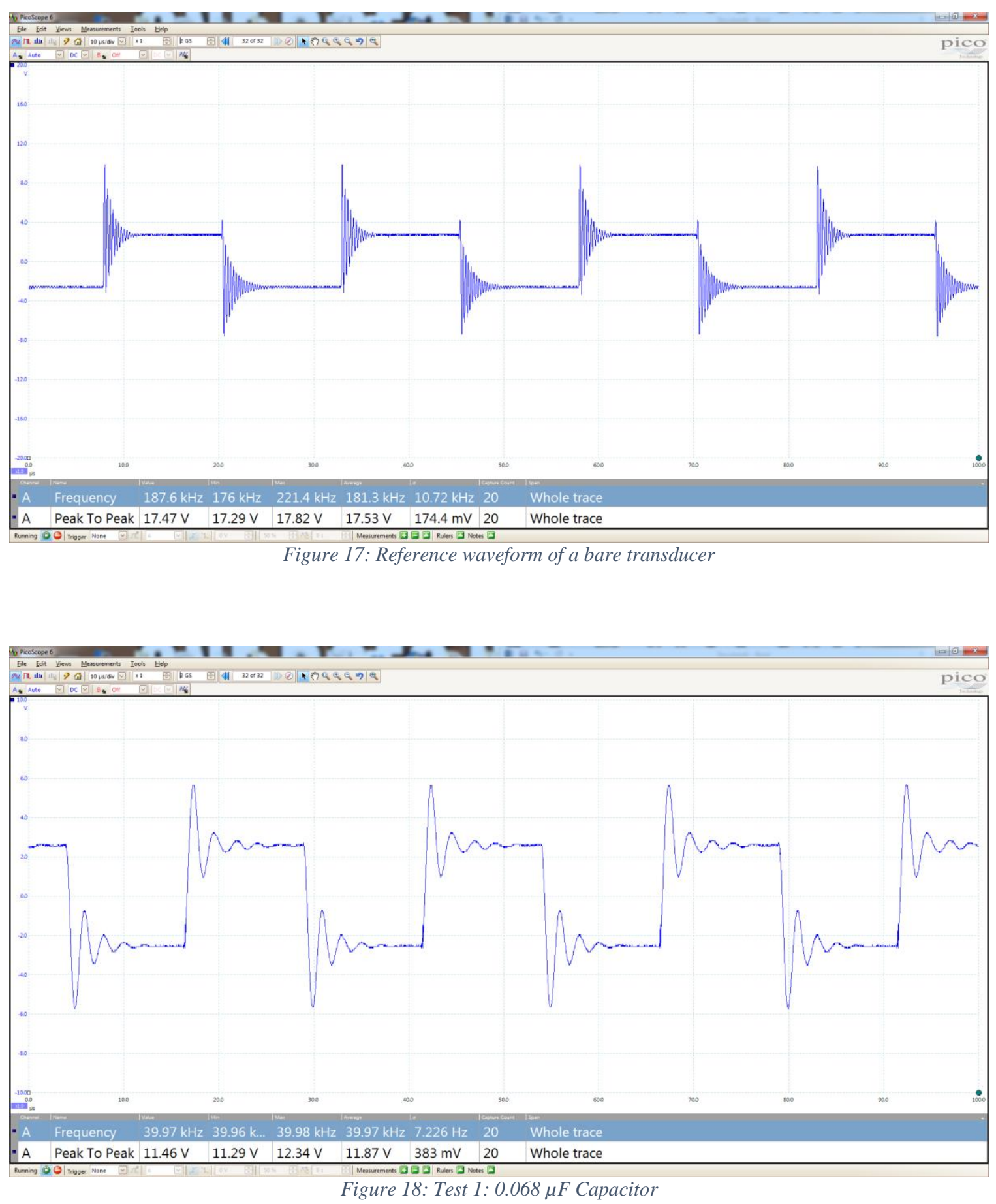

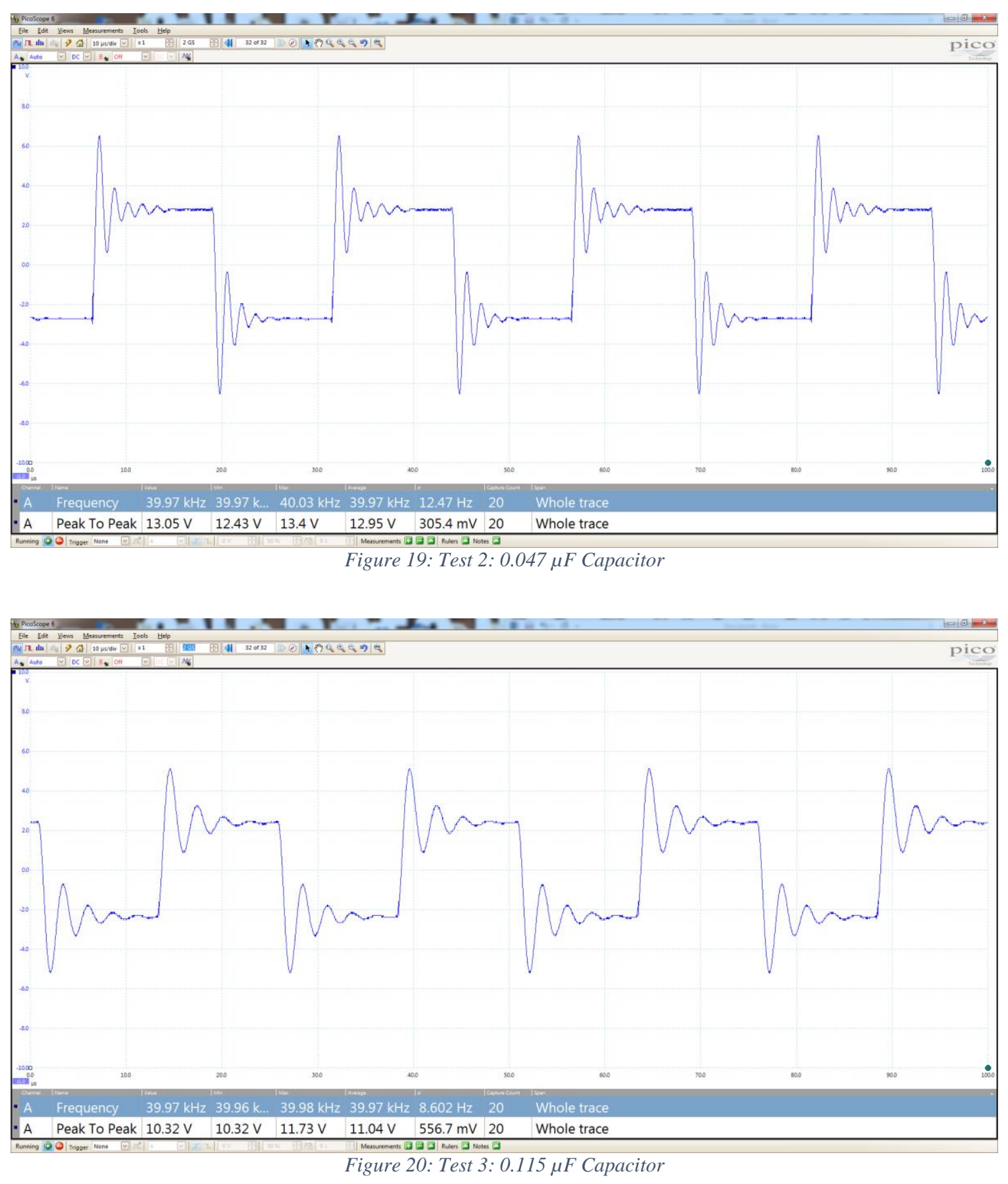


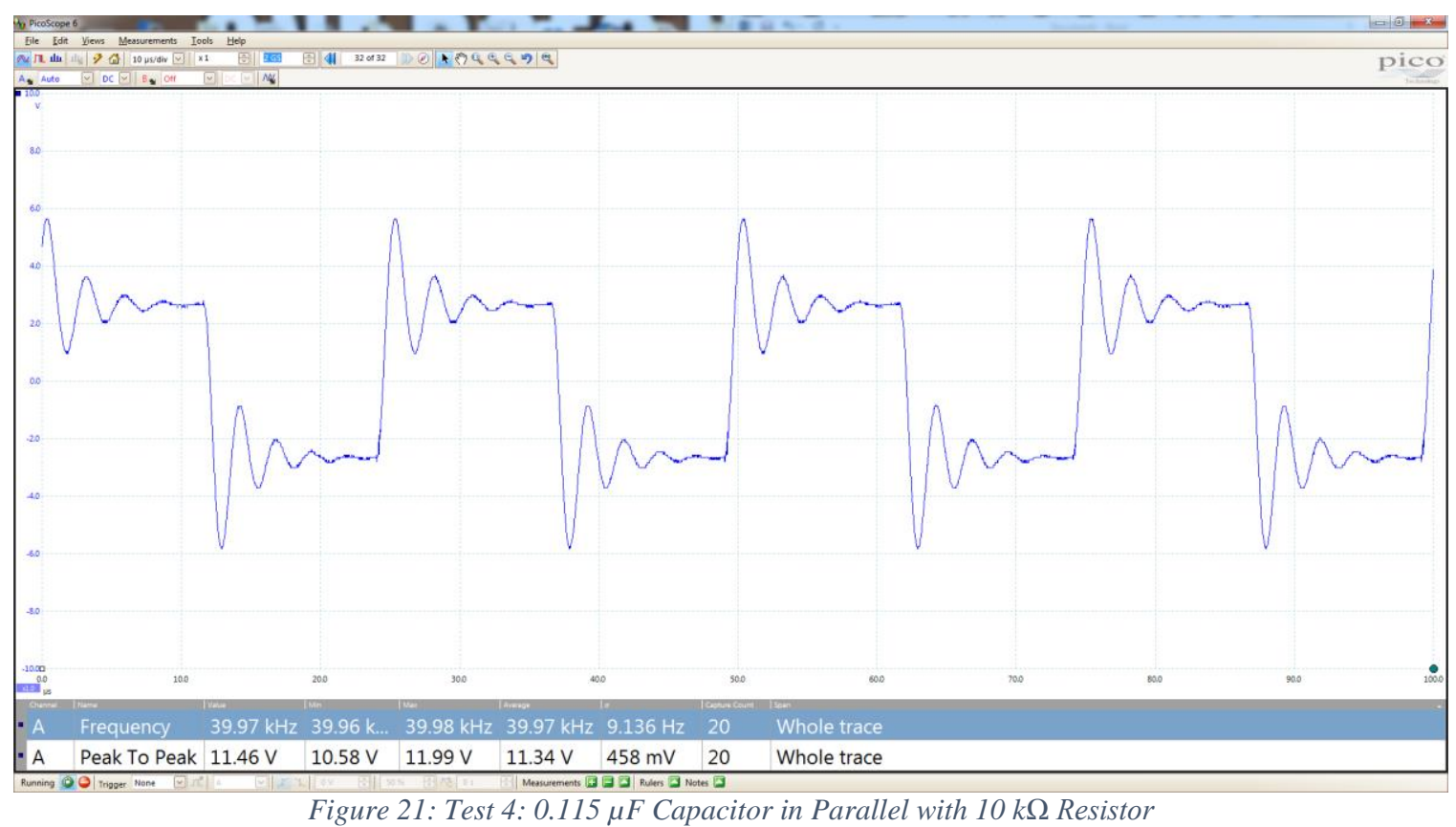

\subsection{Sinusoid Transmission with Commercial Product}

After further consultation about the issue of ringing and the possible ill effects it might have on the experiment, it was noted that the College is in possession of Tektronix AFG2021 waveform generators. The AGF2021 is markedly more accurate than the original homemade kit with the added benefit of clean sinusoid generation. One limit of the AFG2021 that was less than the previous circuitry is it has a maximum peak-to-peak voltage of 9 Volts.

For this test, the apparatus is assembled as described in Section 2.4.3. The AFG2021 was set to generate a $40 \mathrm{KHz}$ sine wave, which is fed to the first transducer and appears as the blue trace in Figure 22 below. The received waveform appears as the red trace below. 


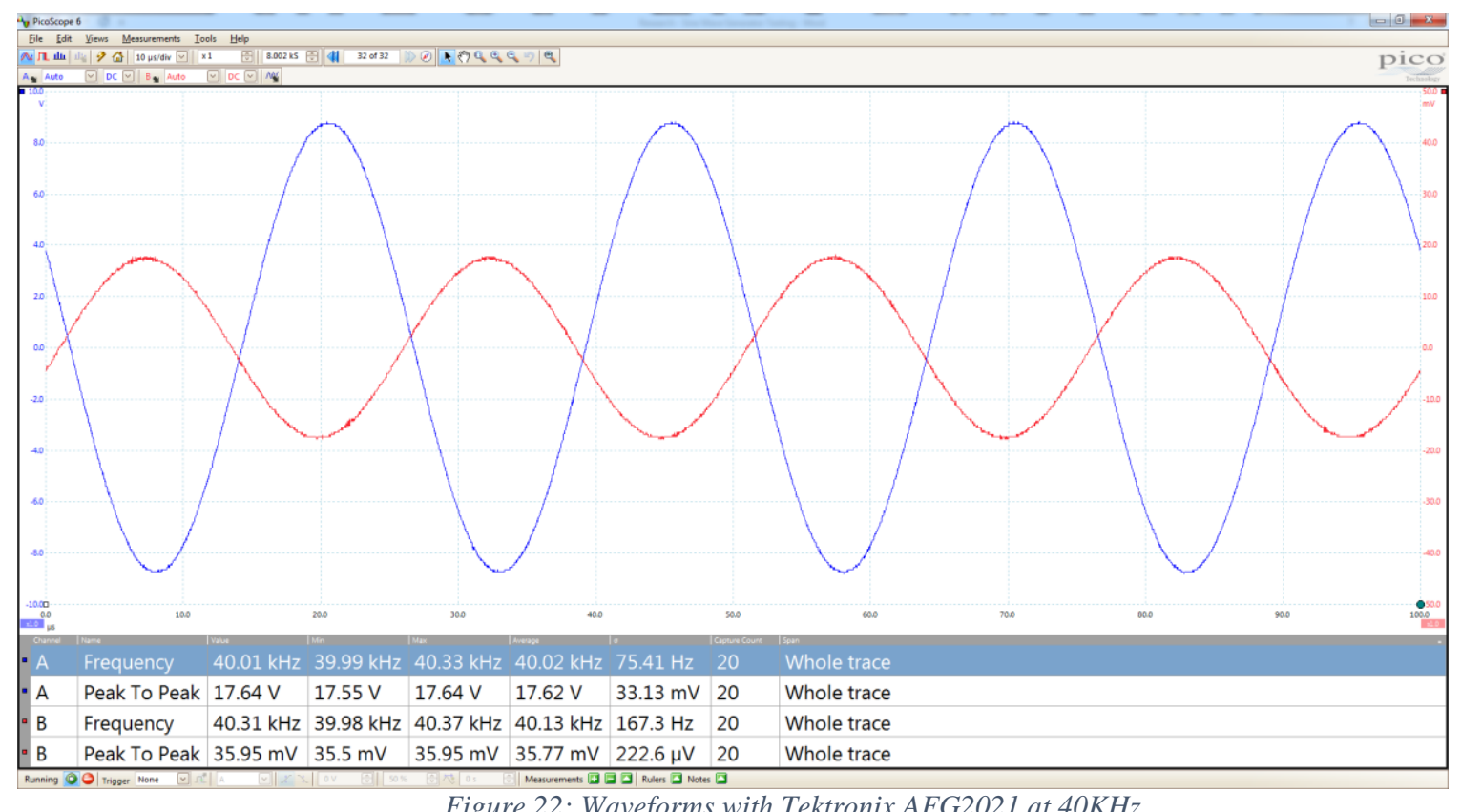

Figure 22: Waveforms with Tektronix AFG2021 at 40KHz.

The result was an extremely clear waveform on both transmission and reception.

Examining the figure above further, there still existed a drift from the desired transmission frequency of 40 kilohertz. Due to drift in the transmission frequency, and an accurate Doppler measurement depending on there being minimal drift, efforts shifted to a transit-time approach, which is believed to be a simpler solution.

\subsection{Initial Time-of-Flight Research}

As an initial test set-up for transit-time experiments, the Texas Instruments TDC1000TDC7200 EVM is examined. The TDC1000-TDC7200EVM is an evaluation module built specifically for Time-of-Flight measurements. The module comes complete with a graphical user interface for configuration of the board as shown in the figure below. Per the User Guide 'The TDC1000 analog-front-end (AFE) is an ultrasonic analog-front-end for Time-of-Flight (TOF) applications operating with ultrasound frequencies up to $4 \mathrm{MHz}$. The TDC7200 is a Time to 
Digital Converter (TDC) that performs the function of a stopwatch and measures the elapsed time between up to five events.' [18]

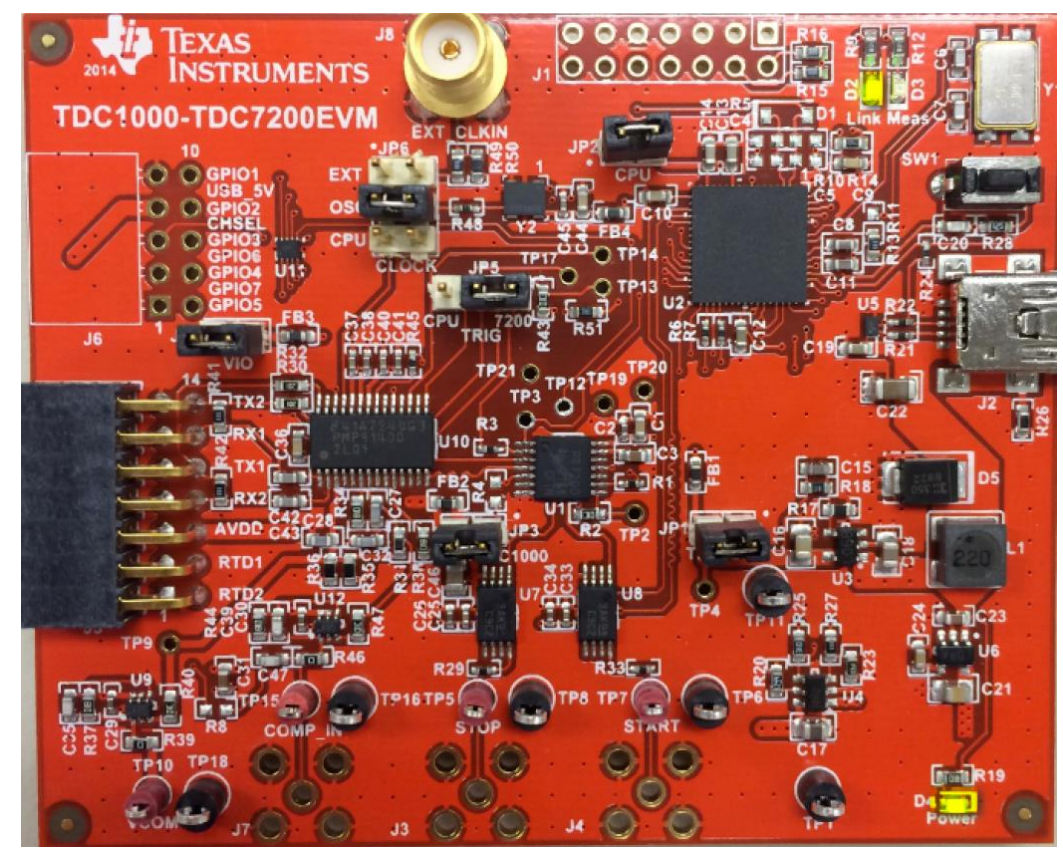

Figure 23: Image of TDC1000-TDC7200EVM [18]

Texas Instruments provides a tool that specifically illustrates the usage of the TDC1000TDC7200EVM with transducers situated across a pipe on a forty-five degree angle as shown in Figure 24 below.

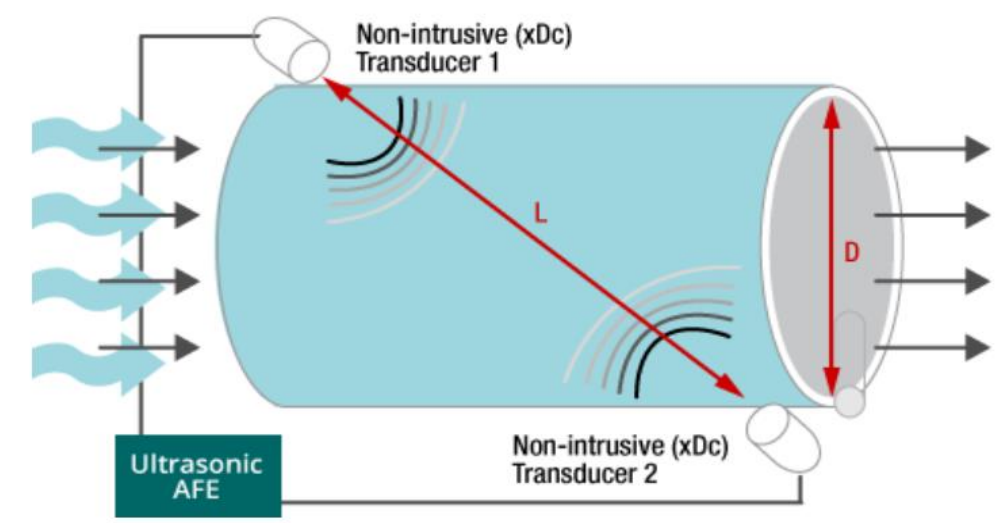

Figure 24: Capture of Texas Instruments Webench Tool Illustrating 45 Degree Transducer Placement [19] 
Prior to testing the EVM, wedges are needed to allow the transducers to be mounted at forty-five degree angles. The first iterations of these wedges are three-dimensionally printed to fit the axial outer diameter of the test piping (shown at the right) and allow the transducers to be affixed to them.

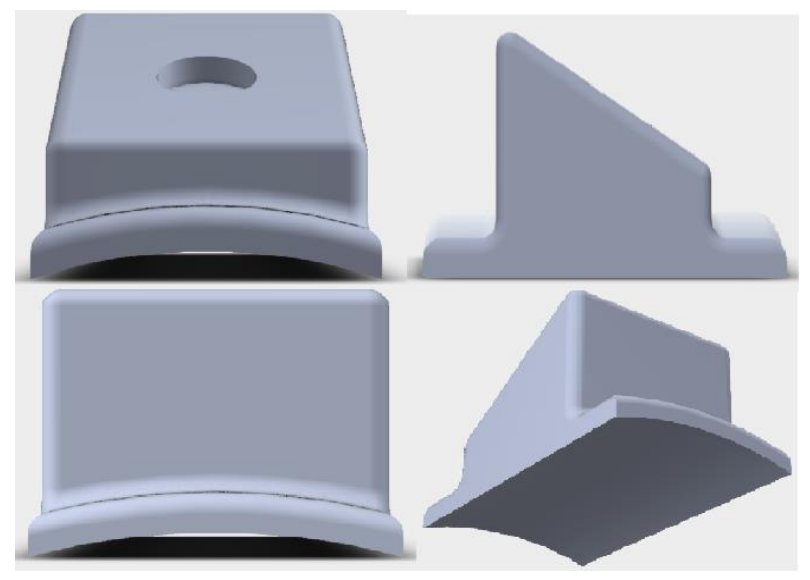

Figure 25: Composite image of a first generation 3-d printed transducer mount.

Being dubious about the acoustic properties of the plastic, it was decided that aluminum transducer mounts should be machined for the least amount of acoustic damping. The resulting transducer mounts were machined from a single block of aluminum with the help of machinist James Hall.

The transducer mounts were situated such that a fortyfive degree angle intersected the pipe and mounted using twopart cyanoacrylate. One Steminc SMD15T21R111WL 1 Megahertz transducer was affixed to each transducer mount using cyanoacrylate. The pipe was filled with water and calibrated in zero flow conditions as shown in Figure 26.

After many adjustments and parameter settings, the EVM would detect zero-flow conditions but would not do so

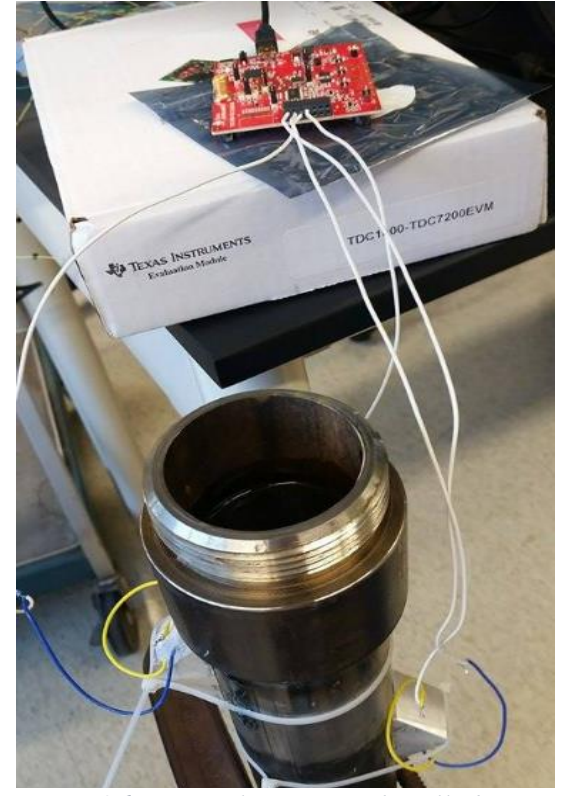

Figure 26: Example setup with milled transducer blocks affixed via cyanoacrylate 
when attached to a fire hydrant. To further investigate, a multi-channel oscilloscope was used to view the signals.

In Figure 27 below, the top channel displays a START signal that coincides with the excitation of the sending transducer. The ultrasonic waves generated and received are shown in the middle channel. Once a configurable threshold has been reached to detect an ultrasonic pulse - and not just an echo - a STOP signal is generated on the bottom oscilloscope channel. The time between the START and STOP signals is the Time-of-Flight through the fluid medium. Multiple tests were done to analyze the echo envelope and find a threshold that would appropriately function with water flowing.

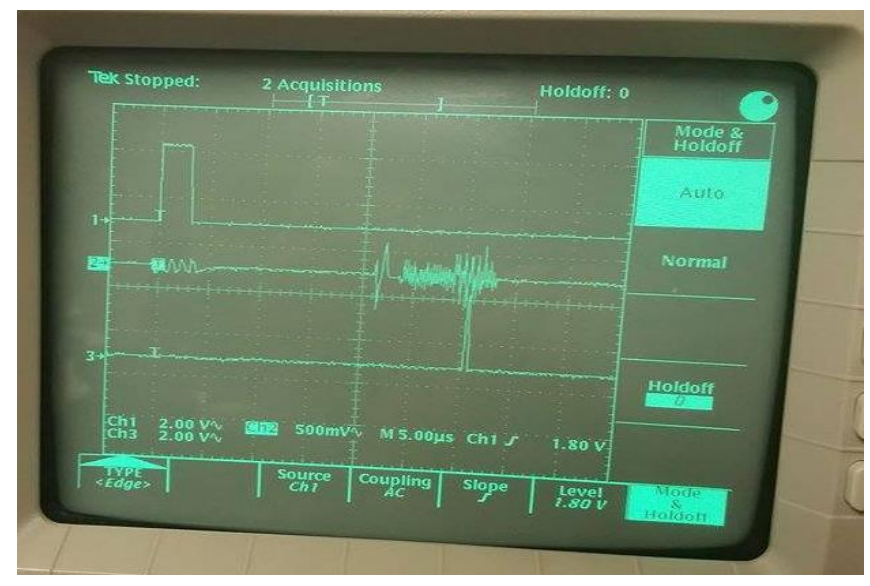

Figure 27: Example oscilloscope output on EVM board

A problem arose with erratic timing when the fluid was flowing. Even at low flow rates the oscilloscope showed multiple triggers, as in Figure 28, that were incorrect. Blanking, a method to ignore input signals for a period of time, was used to eliminate signals outside of an acceptable range. However, the echoes and reverberations made it impossible to blank out the unneeded signals and amplify the desired signals by an amount that did not cause further incorrect readings. 


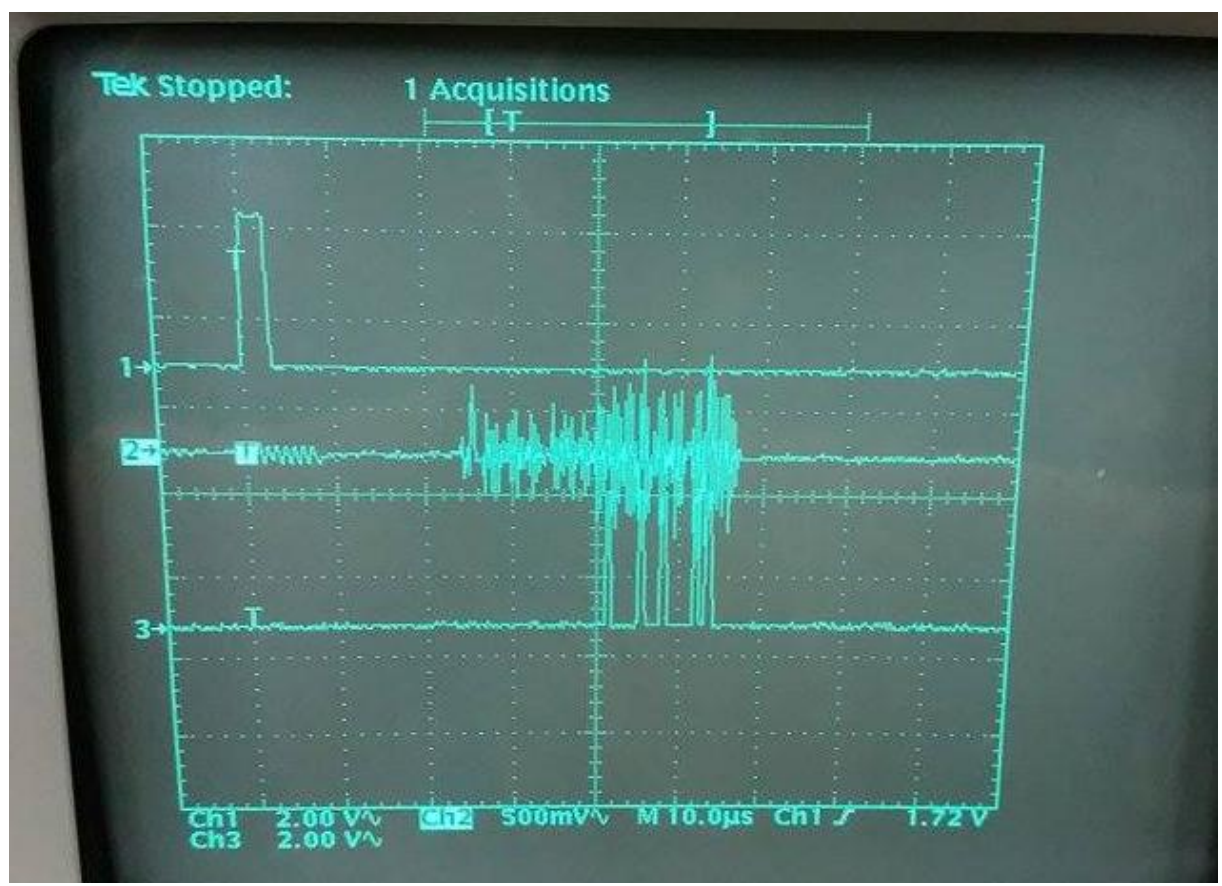

Figure 28: An example of multiple 'STOP' signals caused by increasing gain

The issue can be reduced to the following: Gain could not be increased too high or the noise generated would cause STOP signals to generate incorrectly under no-flow conditions. With the gain set too low, the STOP signals would correctly generate under no-flow conditions but the receiving channel would be masked with noise once flow was initiated.

In consultation with TI support about the issue, they determined that the use of wedges was not supported by their product. While their Webench tool shows transducers mounted at a forty-five degree angle relative to the pipe, they require special hardware to achieve transducer contact with the medium - the hardware is not available in sizes suitable for this project and creating an opening into the pressurized pipe is not desirable as it creates a point of failure for the pump.

To test the effects of a fire truck output on a flowmeter it was determined to move forward with testing a commercial flow meter to determine the feasibility of the project. 


\section{Chapter 3 - Commercial Flowmeter \& Apparatus Experiment}

This section details all equipment used in the flowmeter testing of this experiment. Every item down to the same sections of hose and couplings were reused for all scenarios.

In order to test this experiment on a fire apparatus, without potentially damaging or modifying an existing apparatus, a threaded attachment was machined by a local shop out of steel to serve as a test point on the fire apparatus. The attachment is thirty-six inches long with 2 $1 / 2$ " National Hose male threads on each end and is shown in Figure 29. Due to the nature of the threads, the pipe was cut on a Computer Numerical Control lathe to create the appropriate pitch. The National Hose threads are the American Standard Fire Hose Thread - the standard connection between hose sections and from apparatus to hose. The machined attachment allows the flowmeter to be situated between a fire apparatus and its connecting hose and is shown below.

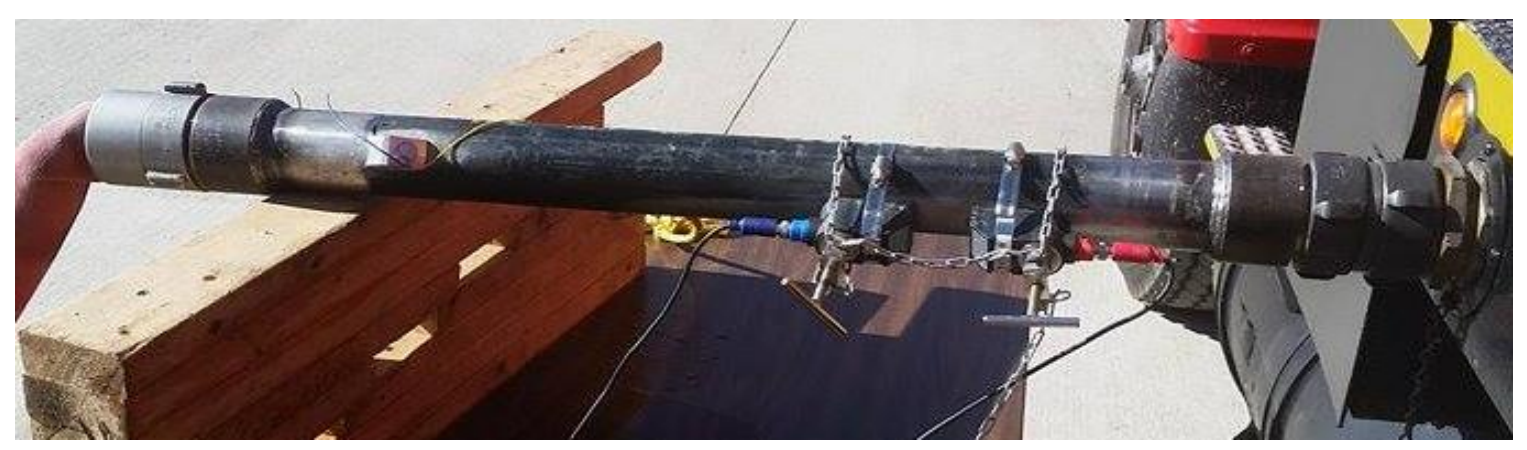

Figure 29: Image of machined adapter hooked to the inlet of a water tender 
The flowmeter, in Figure 30 at the right, used in this experiment is a TDS- $100 \mathrm{H}$ portable ultrasonic flowmeter. Benefits of this flowmeter include onboard logging, which can then be forwarded over a serial connection to a computer for analysis. The meter specifications include linearity of $0.5 \%$, repeatability of $0.2 \%$, and an accuracy of $\pm 1 \%$ at speeds greater than 0.2 meters-per-second. [20] This meter was purchased as the cost to rent similar meters was prohibitively expensive even at an educational rate. The flowmeter is the heart of the experiment.

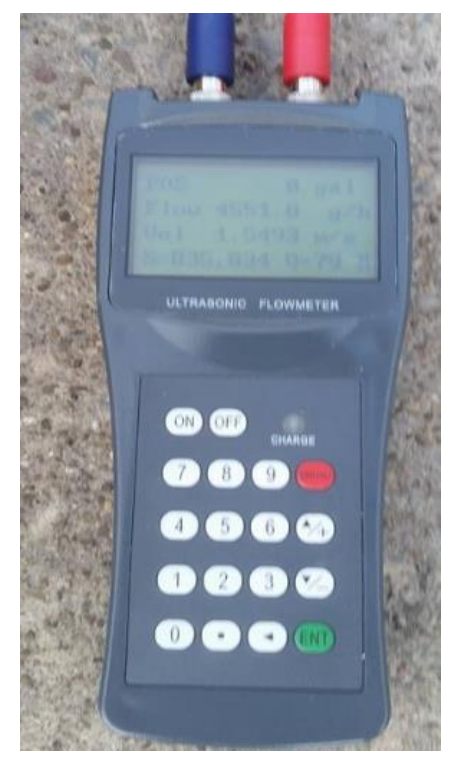

Figure 30: TDS-100H Flowmeter

The use of fire apparatus was granted by a local fire department. The water supply and closed circuit test bed is a water tender with capacity of approximately 2,200 gallons. Also mounted to this apparatus is a centrifugal pressure pump with a $2 \frac{1}{2}$ " discharge rated for a maximum of 208 GPM. The hose used for this test was two twenty-five foot sections of $2 \frac{1}{2} 2$ ” double jacket hose. Additionally, a fire engine was used as a sort of mobile vessel that could be weighed at a local log yard.

\subsection{Flowmeter Setup}

The TDS- $100 \mathrm{H}$ boasts a relatively user-friendly interface that is highly configurable. The driving force behind the usability of the device is the ability to input parameters such as the pipe material, diameter, thickness, transducer mounting method, and fluid type. Once these parameters are entered the meter automatically calculates the distance needed between transducers. 
The steel pipe was sanded by hand to remove a protective outer coating and allow for the best sonic coupling effect. A line was scribed parallel to the length of the pipe to serve as a guide for mounting the transducers in line. The next step was to actually mount the transducers the correct distance apart using an ultrasonic coupling gel and the provided mounting hardware. Once the steel pipe, with transducers onboard, was attached to the apparatus the flow sensors needed to be rotated to account for any possible air that could accumulate in the top of the pipe during low flow conditions which would negatively affect the meter accuracy. Acceptable positions are outlined in Figure 31.

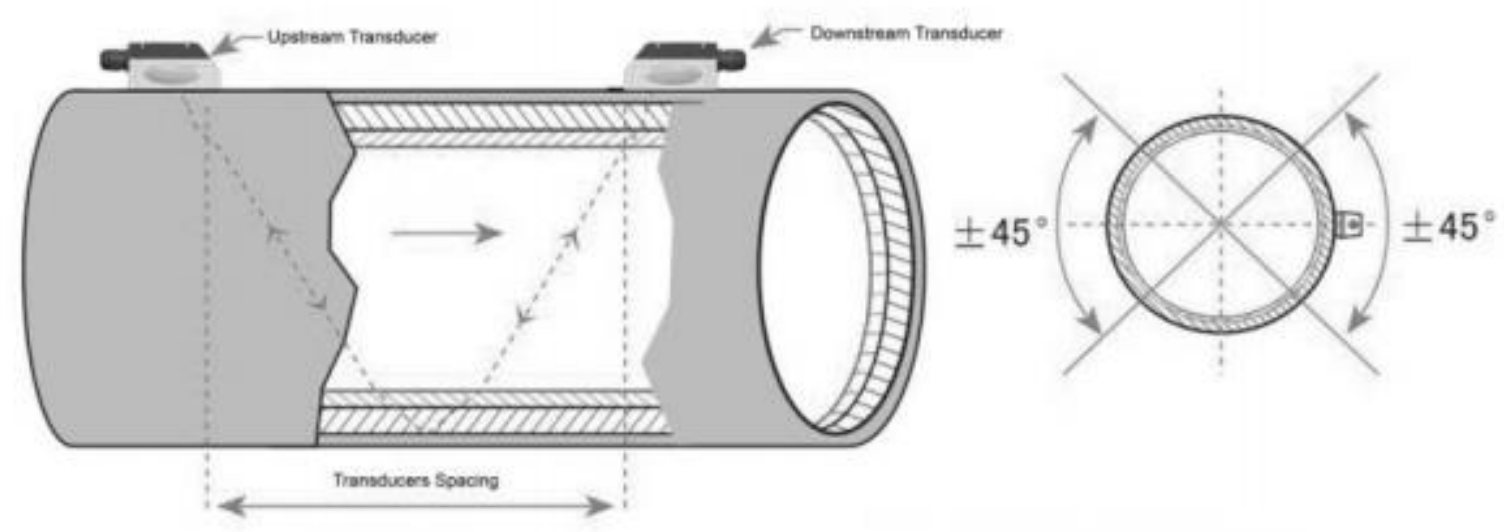

Figure 31: Example of allowable transducer mounting locations [21]

\subsection{Flowmeter Verification}

To test the accuracy of the flowmeter, a vessel of known volume was needed. An approximately one-thousand-gallon fire engine was emptied as far as could safely be allowed without overheating the power-take-off pump. The empty weight of the fire engine was found to be 23,780 pounds. The flowmeter was connected to the truck, and the truck was then connected to a $2 \frac{1}{2}$ " discharge pressure pump hooked to a tank of approximately 2,200 gallons of water with the connecting fifty feet of hose purged of air. The pump was started and the amount of 
time taken to fill the engine until it overflowed was nine minutes and thirty-seven and a half seconds. At this point the fire engine was re-weighed and found to be 32,180 pounds when full of water. The resulting eighty-four hundred pounds of water weight at 8.34 pounds / US gallon works out to a capacity of just over one-thousand seven gallons. Based on this, the actual pump output works out to 1.744 gallons-per-second, or 6278.4 GPH. Examining the flowmeter data, and exempting the initial readings while the valve was closed, the average flowrate reported was 5836.694 GPH. With this information in hand the percent difference between the timed pump flow and the reported flow was found to be approximately $7 \%$. Possible attributions to this error besides flowmeter accuracy include: tolerance of scales used to weigh the apparatus, possible air remaining in the connecting hose, and timing issues related to viewing the water overflow to the bottom of the apparatus.

\subsection{Flowmeter Variable Parameters}

Since the accuracy of the flowmeter was found to be within ten percent at the end of fifty feet of hose, attention turned to testing various pressures and flowmeter locations. The test apparatus used was a 2,200 gallon water tender with a fixed pump mounted onboard. The pump throttle was set and remained the same for all testing. Two twenty-five foot sections of hose were used to create three possible flowmeter locations as detailed in Table 2 below. 


\begin{tabular}{|l|l|}
\hline Position & Description \\
\hline 1 & $\begin{array}{l}\text { Mid-hose, 25 feet from pump } \\
\text { discharge and 25 feet from tender inlet }\end{array}$ \\
\hline 2 & Inlet return to tender, 50 feet from \\
& $\begin{array}{l}\text { Pump discharge } \\
\text { downstream to tender inlet }\end{array}$ \\
\hline 3 & Table 2: Flowmeter test locations \\
\hline
\end{tabular}

Additionally, to achieve a better understanding of the pump characteristics multiple pressures were utilized as detailed in the following Table 3.

\begin{tabular}{|l|l|}
\hline Pressure Index & PSI \\
\hline 1 & 0 (Valve fully open) \\
\hline 2 & 10 \\
\hline 3 & 20 \\
\hline 4 & 30 \\
\hline 5 & 40 \\
\hline 6 & 50 \\
\hline 7 & 82 (Valve fully closed) \\
\hline \multicolumn{2}{|c|}{ Table 3: Pressures used for testing } \\
\hline
\end{tabular}

\subsection{Testing Procedure}

A uniform testing procedure was established for the gathering of data. The procedure was setup specifically to include recording the starting and stopping of the pump. The flowmeter itself was adjusted to record data every second once it was manually triggered. The data recorded and its definition is outlined in Table 4 below. 


\begin{tabular}{|l|l|l|}
\hline Symbol & Meaning & Definition \\
\hline S - or - & Signal Strength & $\begin{array}{l}\text { Refers to amplitude of received ultrasonic } \\
\text { Signals [0-999] }\end{array}$ \\
\hline Q & & Signal to Noise Ratio \\
\hline Flow & Signal Quality & Flowrate in gallons-per-hour \\
\hline V & Flowrate & Fluid velocity in meters-per-second \\
\hline
\end{tabular}

The detailed test procedure is as follows:

1. Connect flowmeter to the test apparatus.

2. Start pump to purge all air from the 2.5 " hose.

3. Run pump to calibrate desired PSI by closing ball valve on inlet and monitoring PSI gauge on pump.

4. Stop pump and wait for fluid to cease flow.

5. Begin flowmeter recording with 10 seconds of no flow.

6. Start pump at the 10 second mark and allowed to run for 3 minutes.

7. Stop pump and allow to wind down for 20 seconds.

8. Stop flowmeter recording at approximately the 3 minute 30 second mark.

9. Download and save data from flowmeter.

10. Erase old flowmeter data.

11. Repeat steps 3-10 for remaining pressures at each test location. 


\section{Chapter 4 - Analysis of Results}

After each test was complete, the data was transferred to a laptop and stored in a text file. A Python script was created to parse the data files and generate corresponding Excel files with selected charts.

\subsection{Position 1 - Mid-hose}

The first position metered was mid-hose twenty-five feet from the pump discharge and twenty-five feet from where the water re-entered the tank. Figure 32, pictured below, shows pump discharge curves as expected with the testing procedure, a ramp-up time followed by a plateau that then terminates in a ramp-down time as the pump is turned off. Perhaps the most interesting part in this first position is that the pump shows a higher discharge rate under a load of 10PSI than it does with no load. Accordingly, as the pressure was increased for each successive test the pump discharge became more linear at operating speed and showed less variation in pump rate. During the final test at this position, it was also determined that the pressure pump had a maximum pressure of approximately 82 PSI when pumping against a closed ball valve at the trunk inlet. The pump maintains this pressure even when powered off, indicating the presence of a one-way valve in the pump body. 


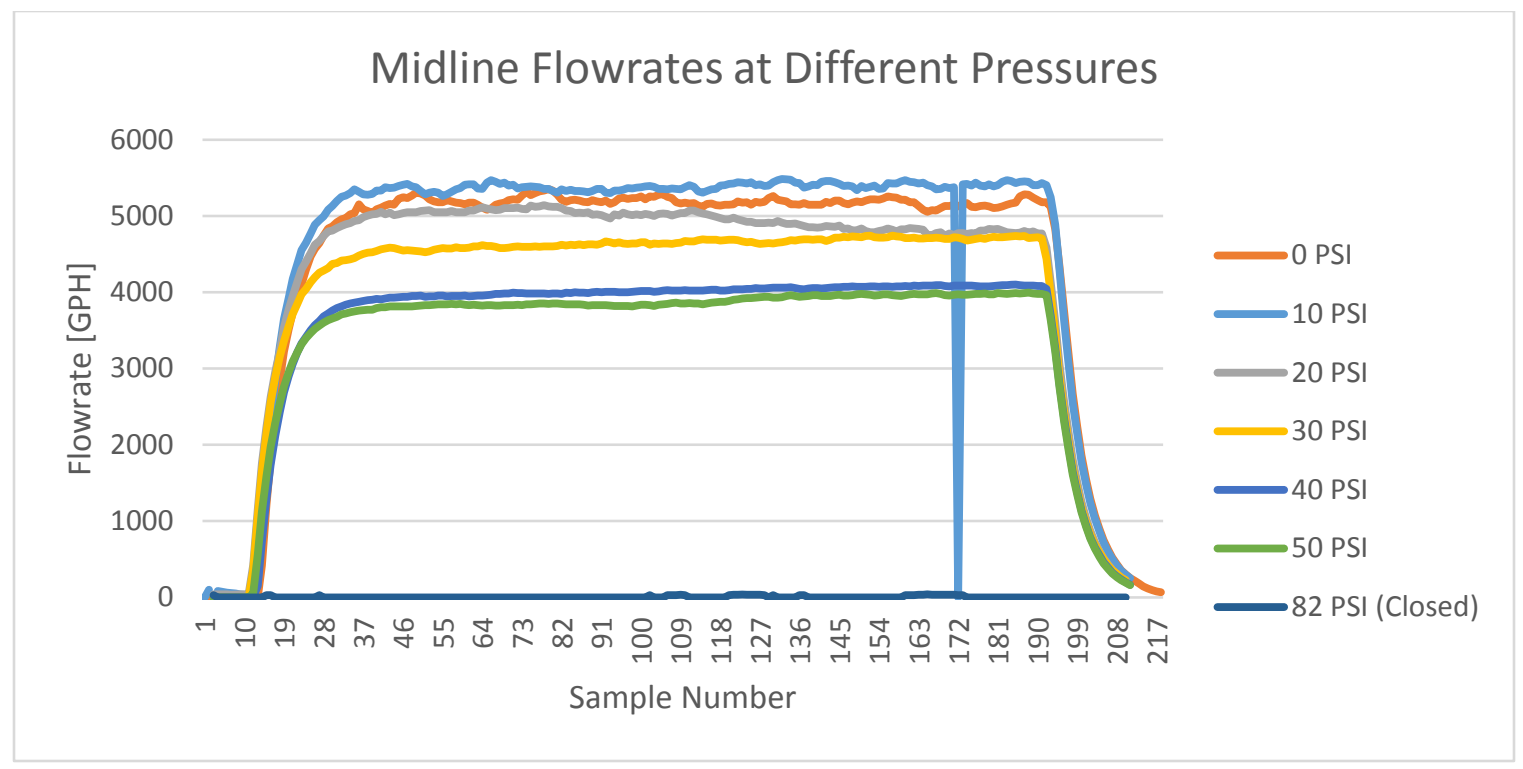

Figure 32: Midline Flowrates at Different PSIs

Further analysis of the data located in Figure 33 at this location shows that the Signal-toNoise Ratio [Q] tends to increase as the flowrate decreases. As a control, when pumping against a closed ball valve the flowrate shows predominately as zero gallons while the signal quality is in the highest twenty-five percent.

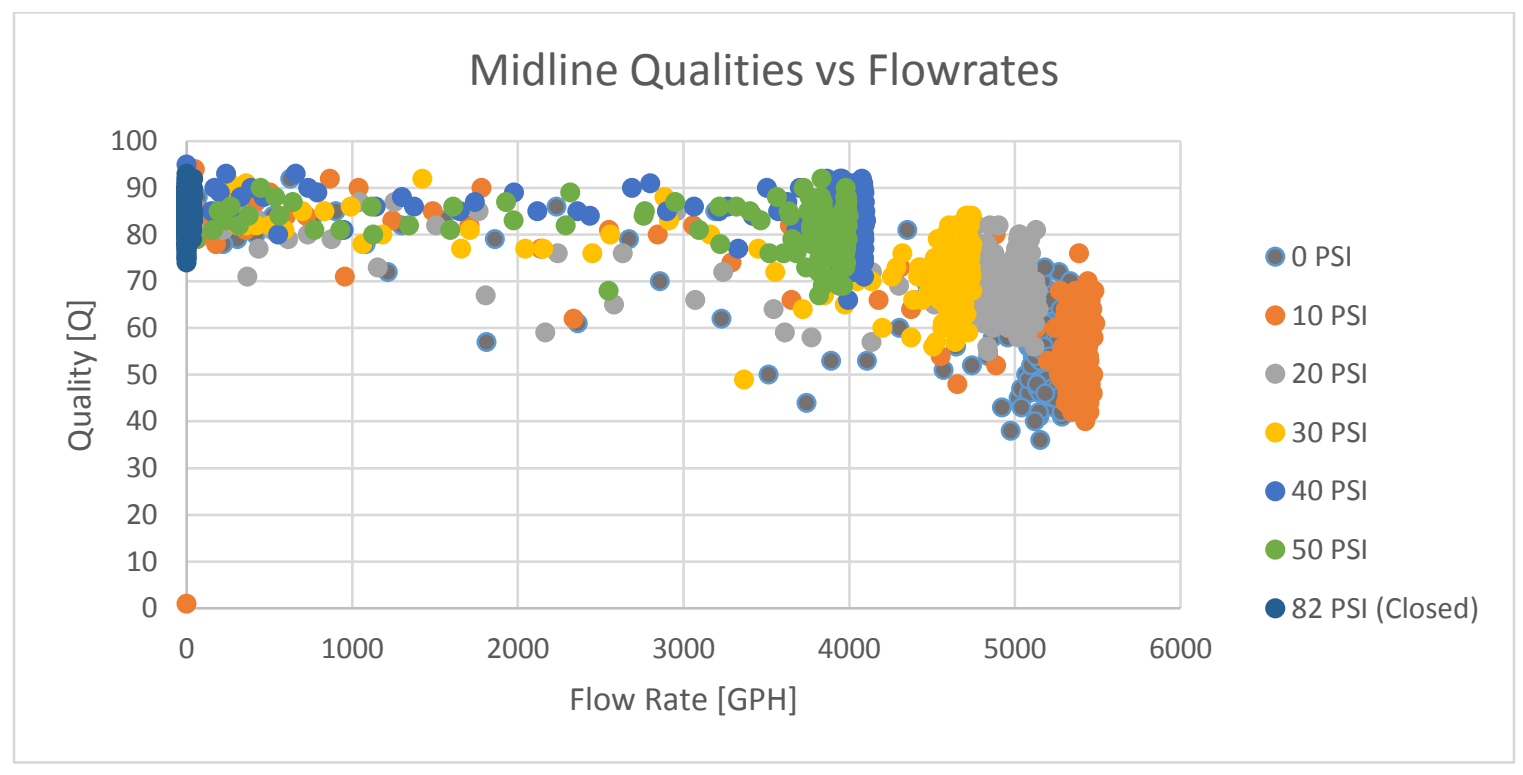

Figure 33: Midline Qualities vs Flowrates 


\subsection{Position 2 - Tank Inlet}

The second position tested was with the flowmeter fifty feet from the discharge of the pump, at the tank inlet. This position places the flowmeter further from potential turbulence caused by the pump than the previous position. Examining Figure 34 below again shows that the pump performs better with a slight pressure of 10 PSI applied. The general pattern of overall flowrate reduction as pressure is increased is predominant. A noted difference in this data is that in Position 1 the GPM for 40 PSI and 50 PSI were very close - but in this position 50 PSI is showing a higher flowrate than 40 PSI

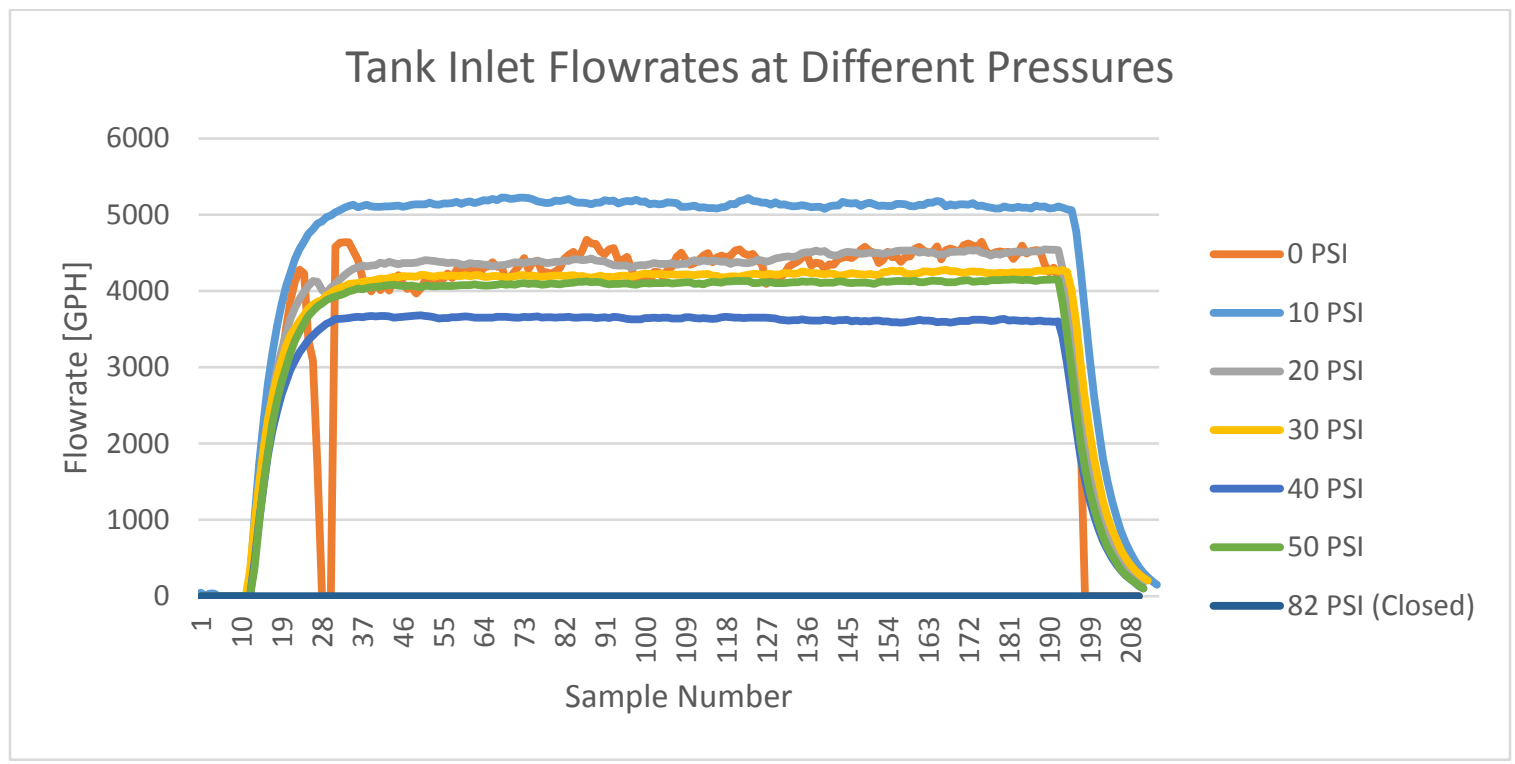

Figure 34: Inlet Flowrates at Different PSIS 
Figure 35, shown below, indicates that the signal quality tends to increase as flowrate decreases. At this position, the flowrates appear to be measured with less variance once pump speed is established. Possible attribution to a lack of variance could be the distance from the pump.

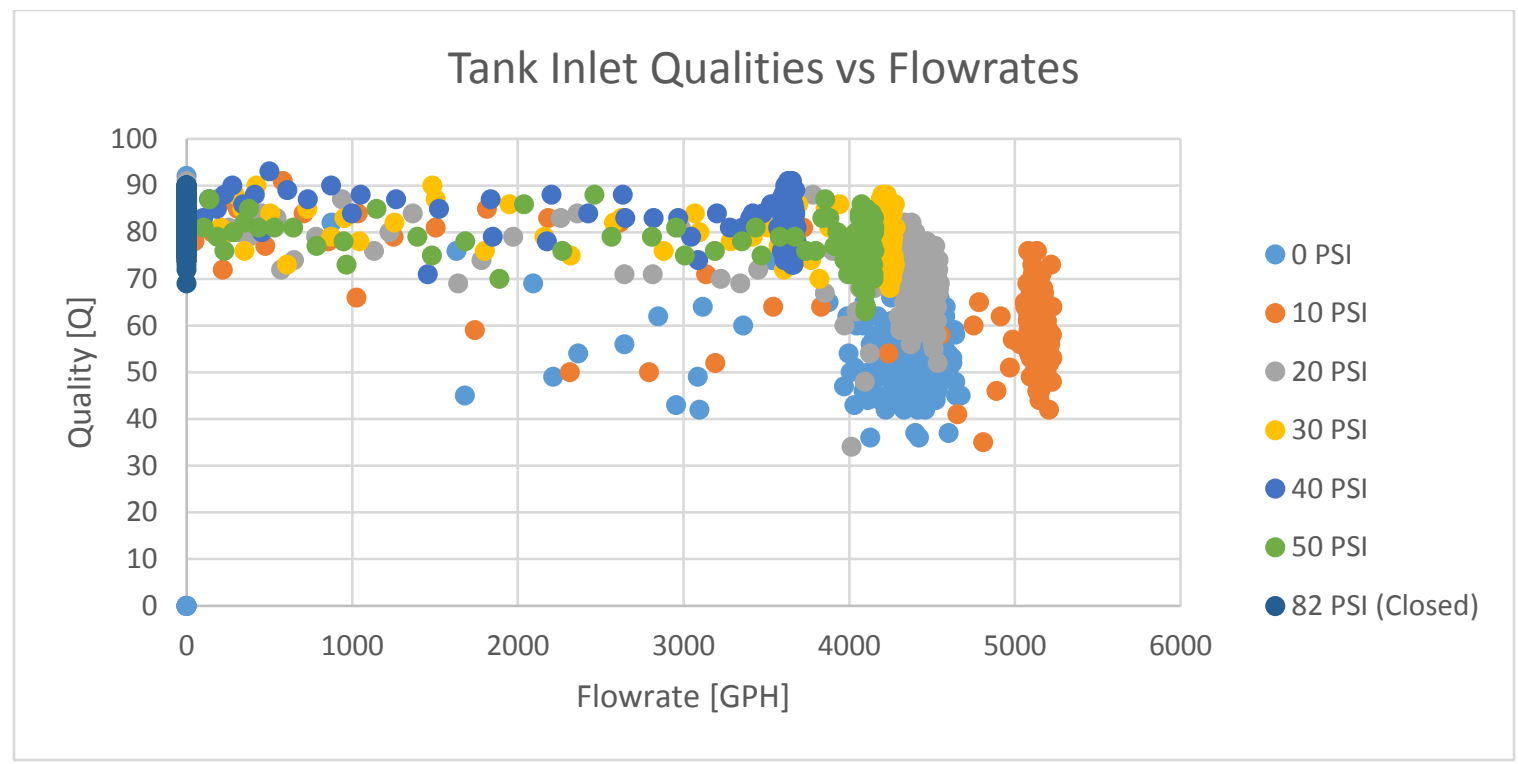

Figure 35: Inlet Qualities vs Flowrates

\subsection{Position 3 - Pump Discharge}

The third position in the original test was to apply the flowmeter directly to the output of the pump discharge. This is the ideal location for the flowmeter to function. As shown in Figure 36 below, at this location the data became unreadable and highly corrupted. The only measurement that performed as expected was the final position with the ball valve closed at the tank inlet - it correctly reads as zero flow. The other numbers vary to a great degree - including even negative flowrates. While the TDS-100H does distinguish between positive and negative flowrates, negative flowrates were an unexpected result. 


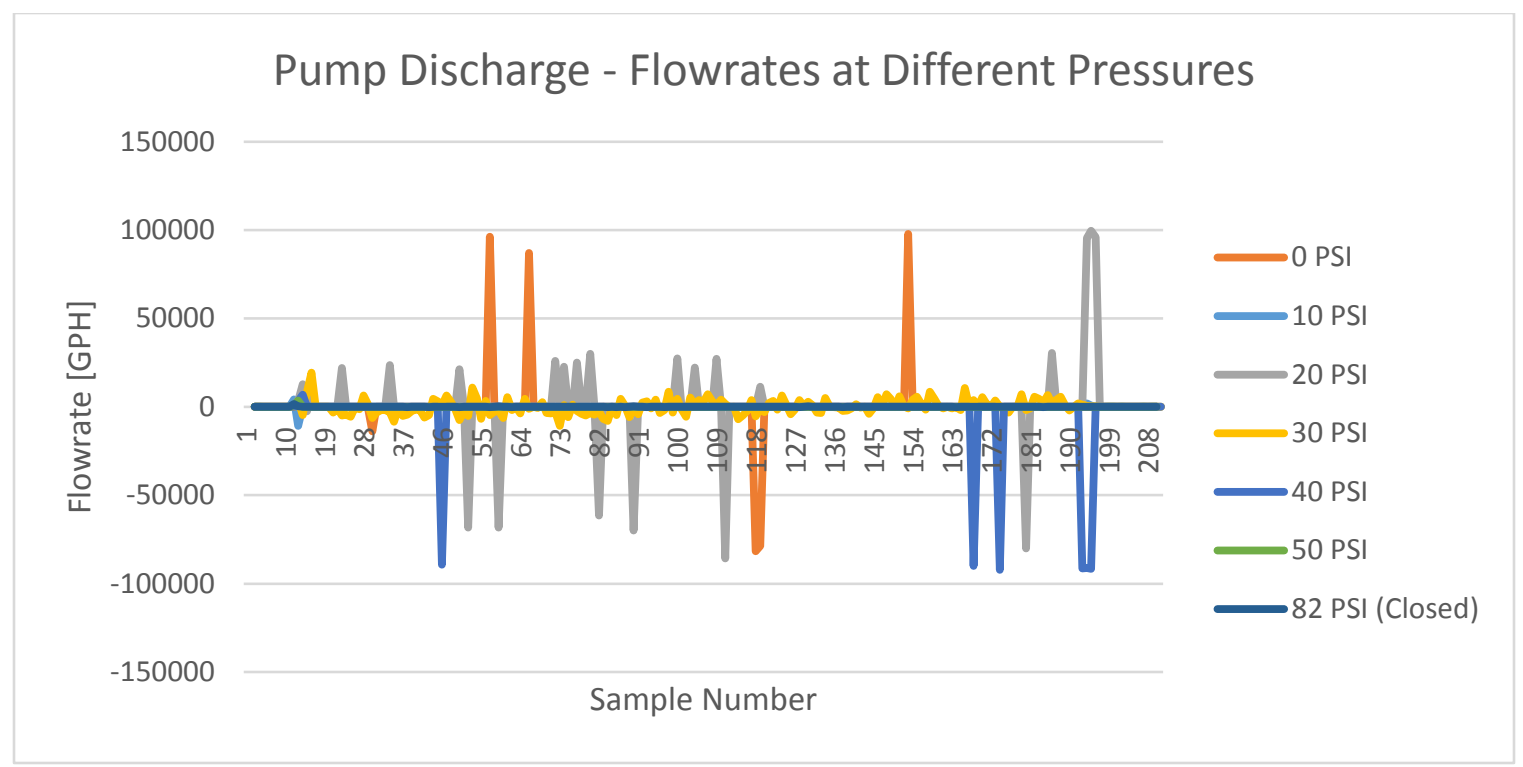

Figure 36: Discharge Flowrates at Different PSIs

Similarly, the signal quality in Figure 37 only appears to be useful when the ball valve is closed and no flow is present. This data also proves to be of little help but, notably, the signal quality is extremely poor at 50PSI but makes a large jump when the valve is closed.

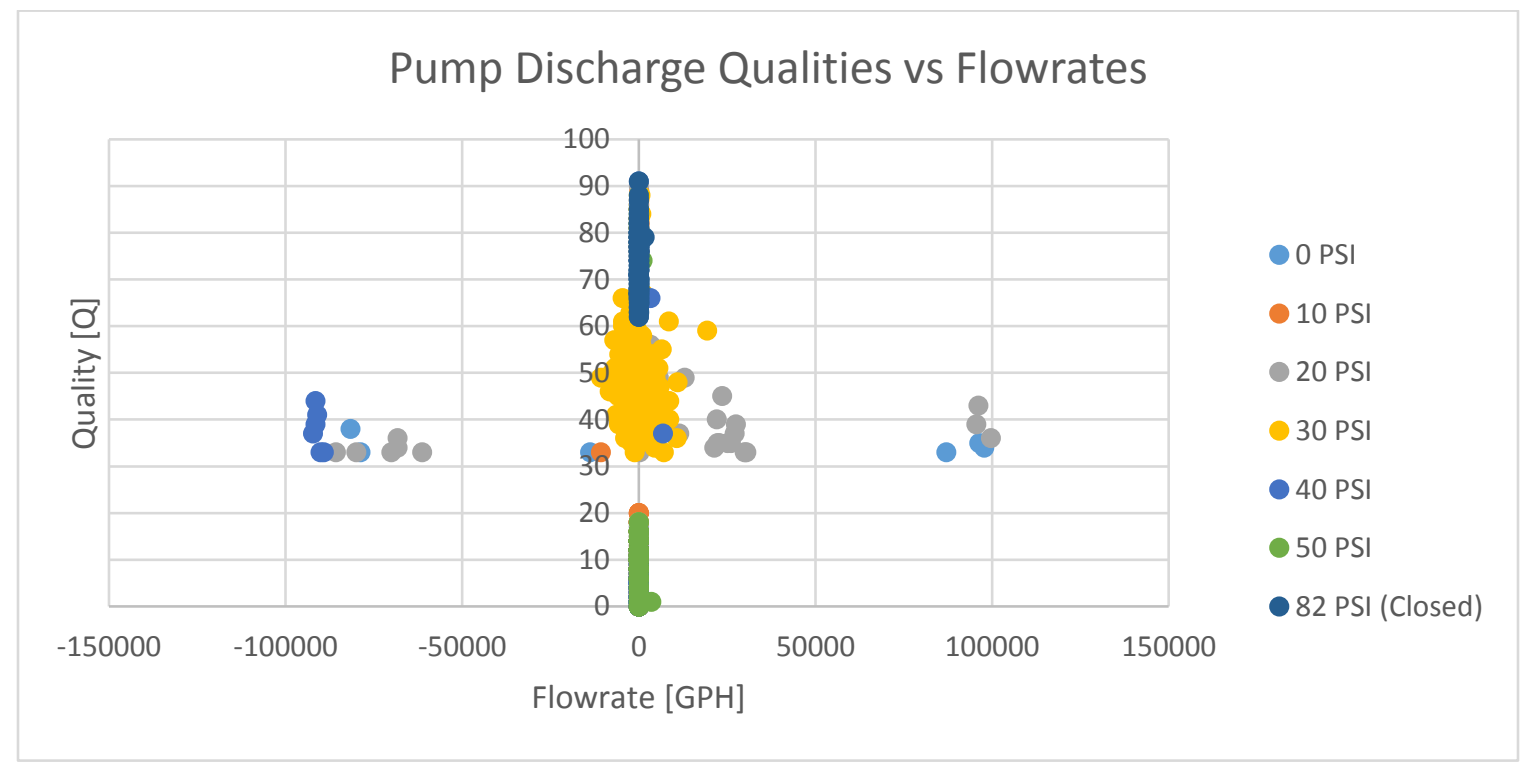

Figure 37: Discharge Qualities vs Flowrates 
With the results from the previous test in hand it appeared the use of a flowmeter directly at the discharge of a pump was problematic. After some consideration, it was concluded that a possible issue could be the mechanical operation of the pump. The operation could create vibrations picked up by the transducer as sound. To test this theory, the transducer was partially decoupled from the pump by means of a small semi-rigid rubber hose normally used for suction. The results were not repeated for all pressures as the stability of the connections to the pump via hose clamp was uncertain. The results shown below in Figures 38 \& 39, while not accurate flow results, show a vast difference compared to the same data without the damping coupler attached. Improvements also appeared to be present in the signal qualities.

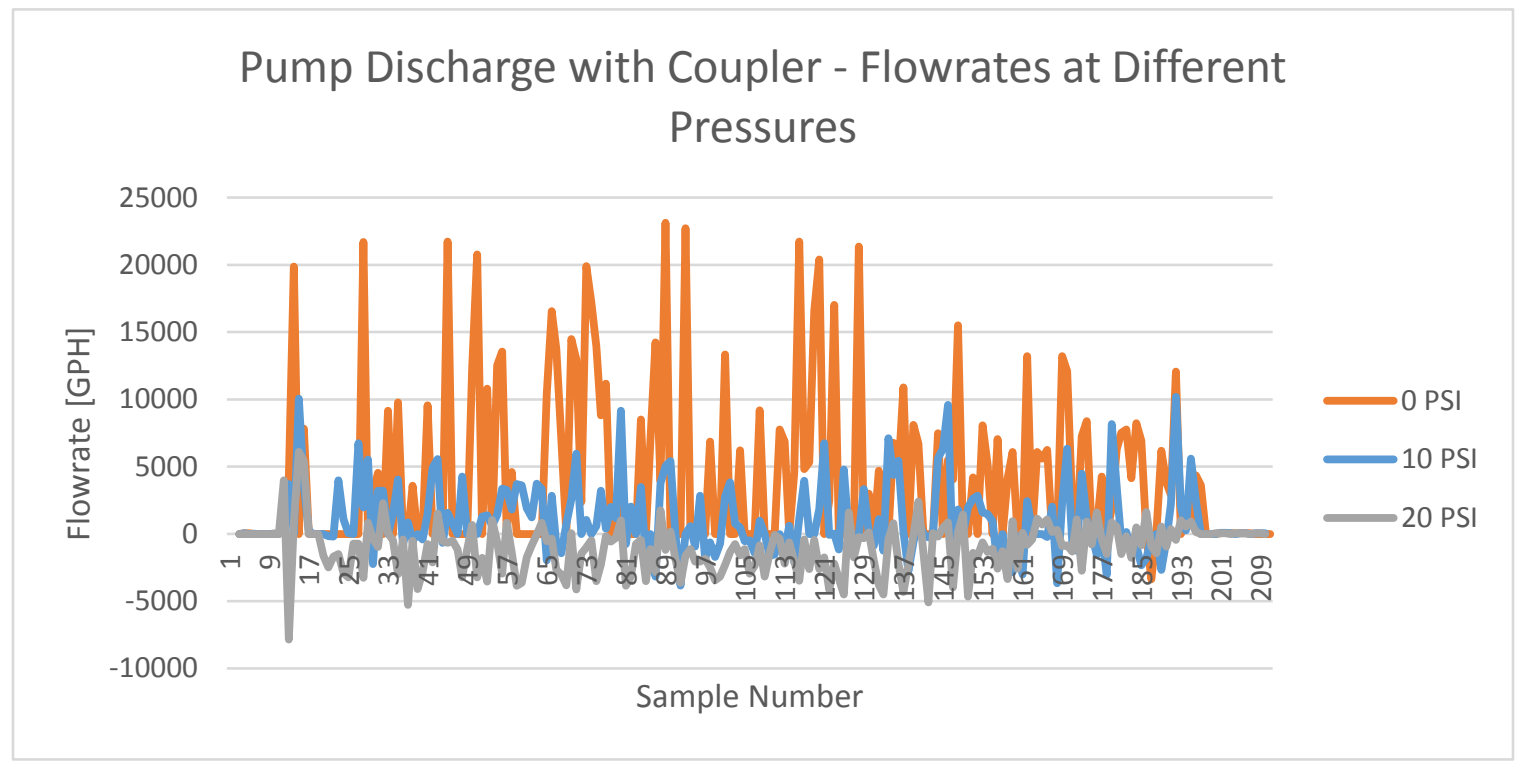

Figure 38: Flexible Coupling Discharge at Different PSIs 


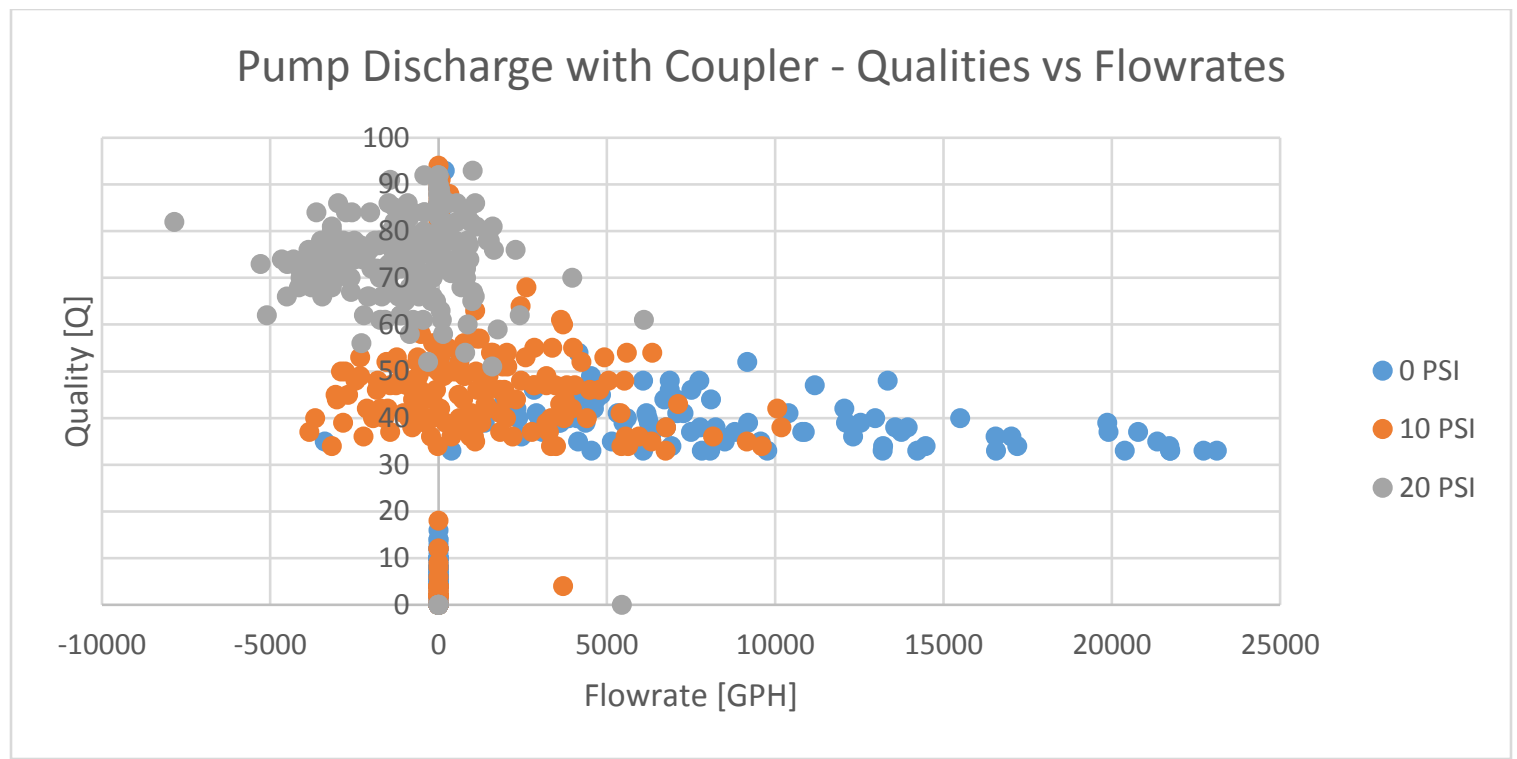

Figure 39: Flexible Coupling Qualities vs Flowrates

\subsection{Further Assessment of Vibration Data}

To get a better sense of the effects caused by the damping rubber coupler, charts from the rigid attachment to the pump discharge were modified to remove pressures that were not tested with the rubber coupler. Additionally, the axes of the charts utilizing the damping coupler were adjusted to match the range from the rigid connection. Figures 40 and 41 are displayed below, one above the other, for comparison. 


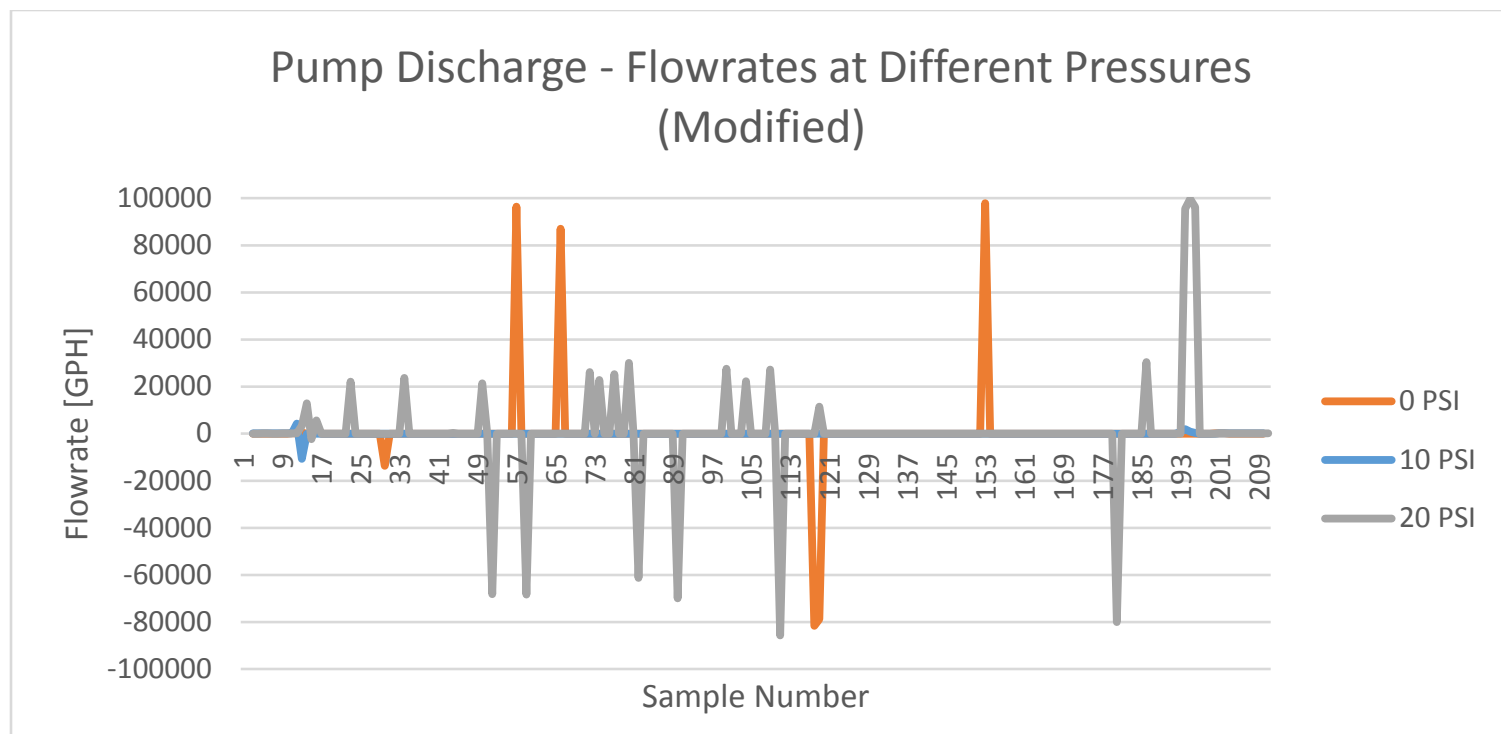

Figure 40: Modified Flowrate Chart

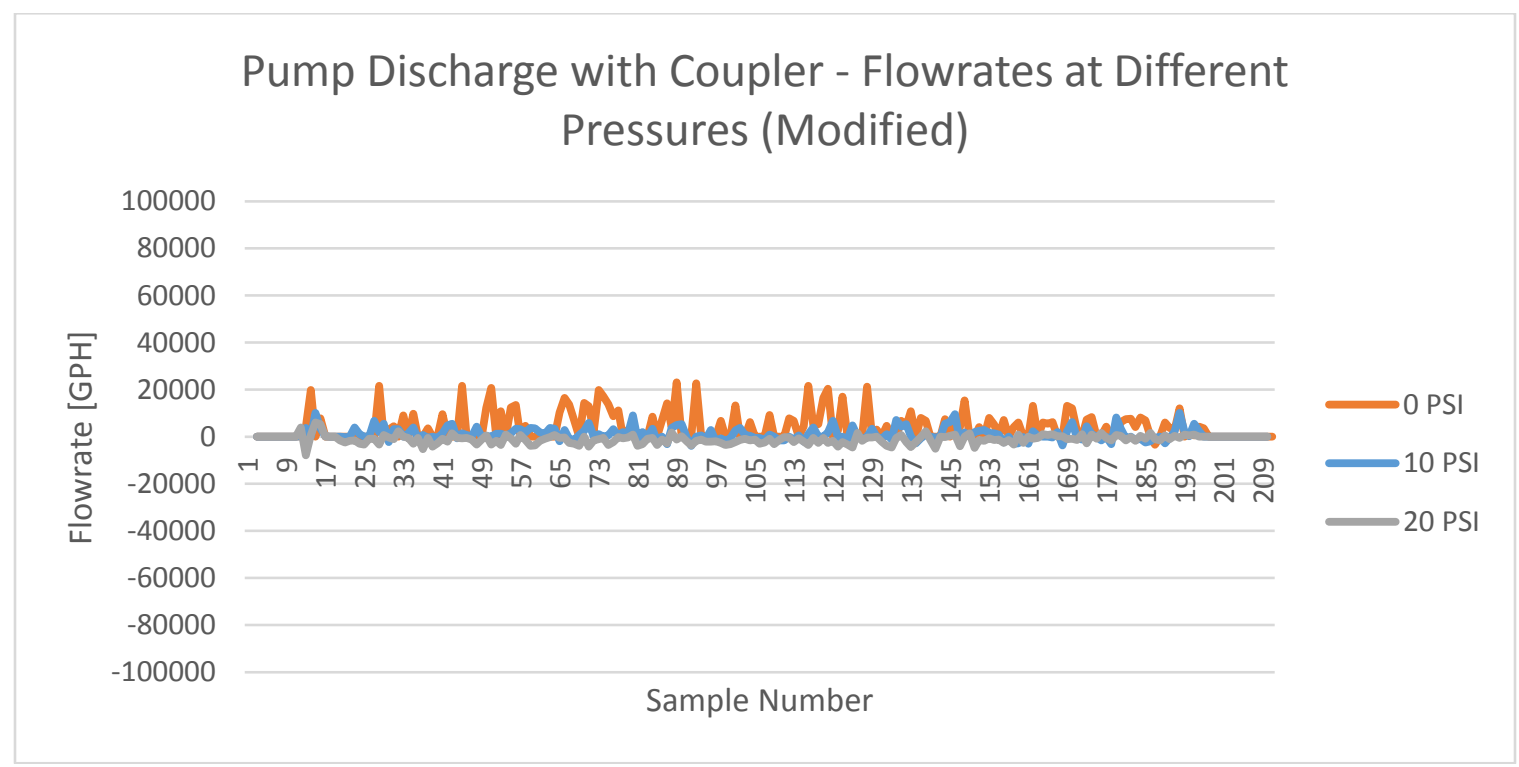

Figure 41: Modified Flowrate Chart (With Coupler)

Similarly with signal qualities, the negative flowrates were essentially nonexistent once a damping coupler was added. Examining the chart in Figure 42 appears to show relatively mirrored flowrates around the $\pm 100,000 \mathrm{GPH}$ range. Once the coupler was induced, in Figure 43, these mirrored flowrates ceased to appear in the data. This seems to indicate the presence of 
rigid connection to the pump discharge induces a harmonic vibration along the length of the test attachment.

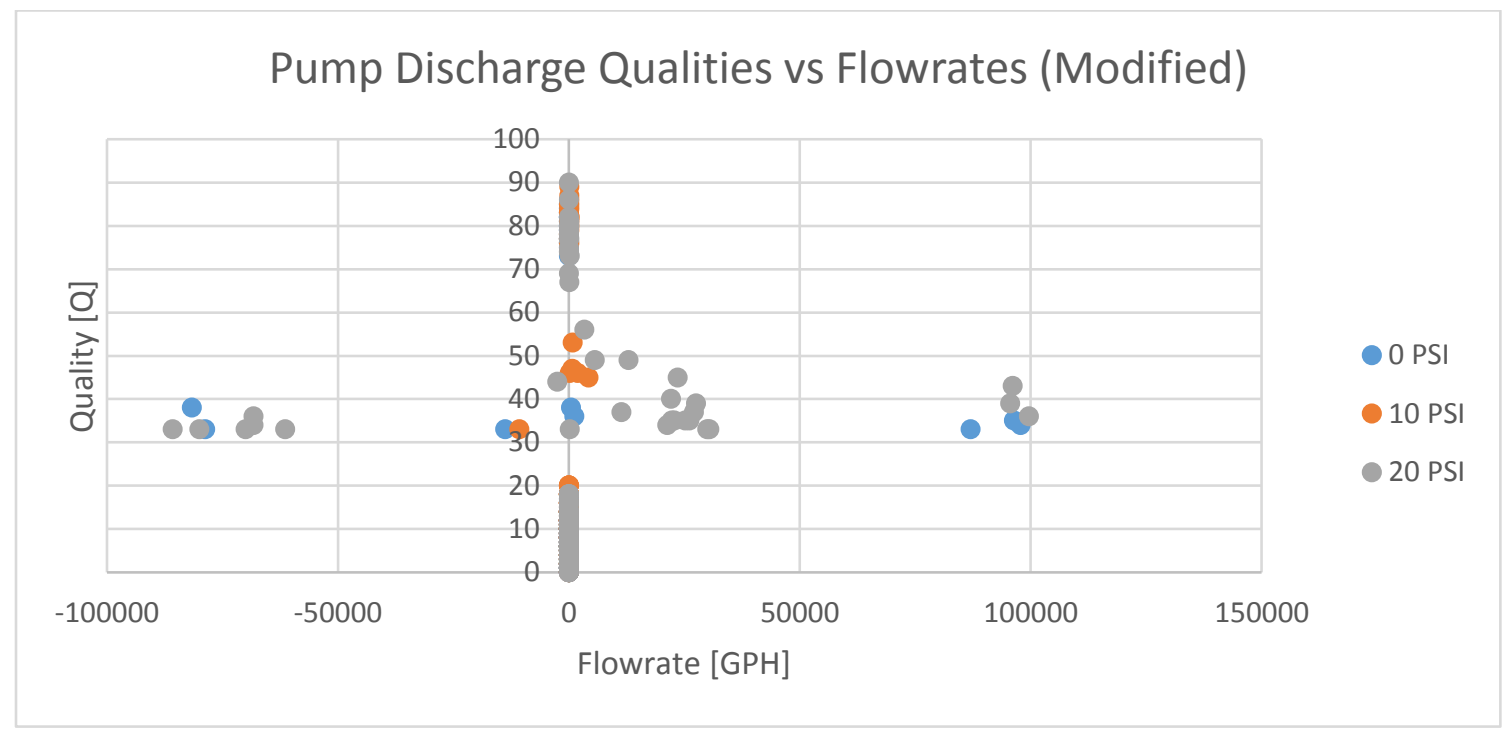

Figure 42: Modified Qualities Chart

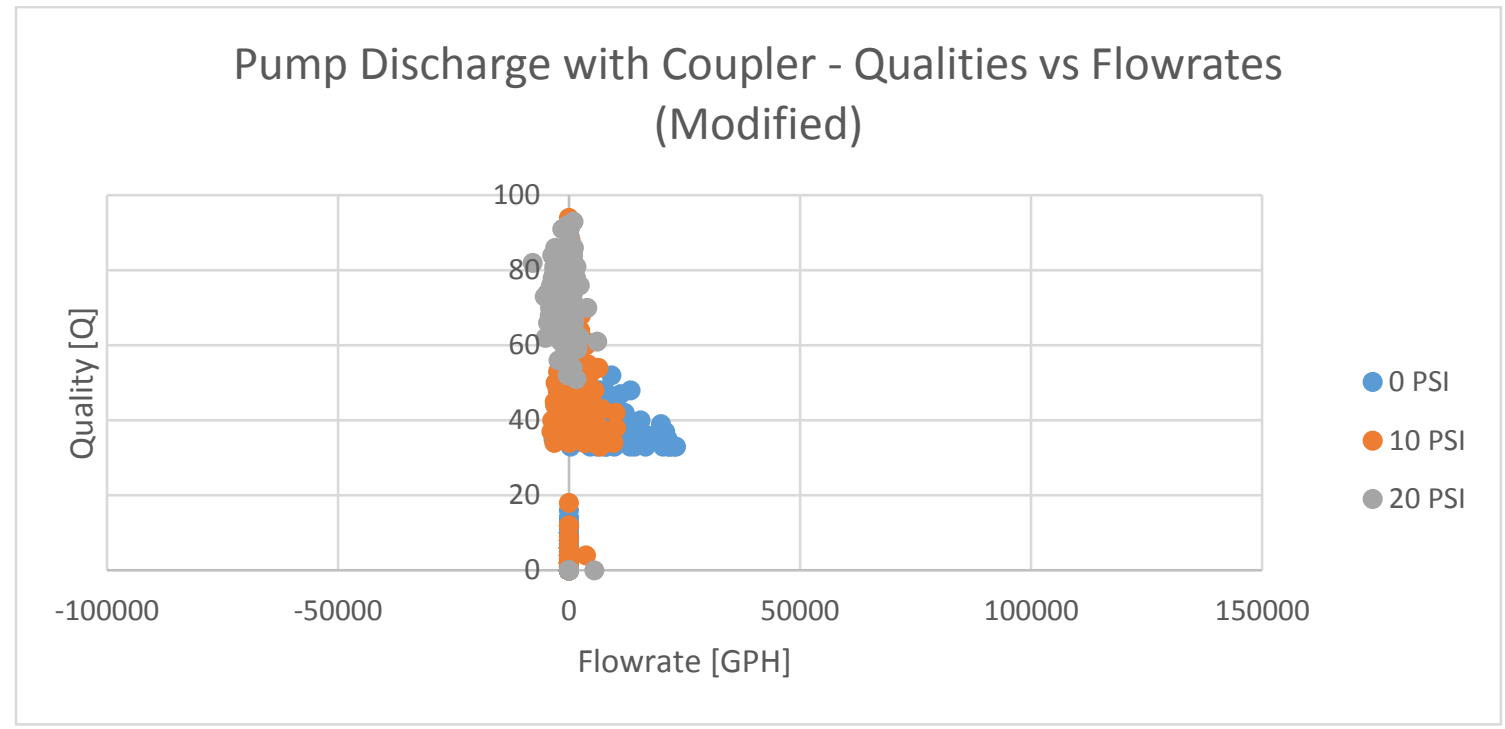

Figure 43: Modified Qualities Chart (With Coupler) 


\section{Chapter 5 - Conclusion \& Future Work}

\subsection{Conclusion}

In conclusion the applications of ultrasonic flowmeters to fire apparatus is promising. The ultrasonic flowmeter performed well at down hose distances of twenty-five feet and fiftyfeet from the pump discharge. When placed directly at the pump discharge the data was very corrupt. This is likely as a result of being rigidly coupled too closely to the pump itself and inducing vibrations in the test apparatus which disturb the velocity readings.

In conclusion, the data seems to indicate that the presence of rigid coupling to a pump induces an undesirable vibration in the testing apparatus. In order to successfully create a working Time-of-Flight flowmeter that does not require modification of a fire apparatus's pump manifold, it appears a filter would need to be built and applied to the flowmeter circuitry. Prior to building a filter, the vibrations of the pump and engine would need to be well-characterized across a wide range of operating pressures and engine RPMs. With this data, a flowmeter and associated filter can be calibrated to a specific apparatus's engine and pump characteristics to reject data outside of the desired frequency range. It may also be necessary to actively adjust the frequency at which the flowmeter functions to avoid conflicts with pump and engine noise.

\subsection{Future Work}

Future work relating to this project will first require a method of characterizing vibrations generated by the fire apparatus. Once characterized, an appropriate method for filtering out the noise will need to be developed. The method of filtering out the vibrations is likely to become an exercise in the realm of digital signal processing. The digital filter will also need to be able to make adjustments in real-time as the vibrations will change with pump speed, engine RPM, and 
possibly tank volume. The presence of a digital signal processor would also be important for the ease with which the filters could be calibrated to individual trucks. Once this process was built and working, the equipment would need to be tested on a wide range of manufactured pumps to ensure accuracy and repeatability. 


\section{References}

[1] W. Maybee, "Key Dates in Fire History," [Online]. Available: http://www.nfpa.org/research/reports-and-statistics/key-dates-in-fire-history.

[2] "National Fire Protection Agency," [Online]. Available: http://www.nfpa.org/news-andresearch/fire-statistics-and-reports/fire-statistics/the-fire-service/administration/us-firedepartment-profile.

[3] "United States Fire Administration," May 2007. [Online]. Available: https://www.usfa.fema.gov/downloads/pdf/publications/fa-310.pdf.

[4] COMMITTEE ON PUBLIC WATER SUPPLY DISTRIBUTION SYSTEMS: ASSESSING AND REDUCING RISKS, "National Academies Press," 2006. [Online]. Available: https://www.nap.edu/read/11728/chapter/3\#20.

[5] "NFPA 1901: Standard for Automotive Fire Apparatus," 2009. [Online]. Available: http://www.nfpa.org/codes-and-standards/document-informationpages $?$ mode $=$ code $\&$ code $=1901$.

[6] C. Cutright, "Pierce Water Level Gauge," 2017.

[7] P. J. LaNasa and E. L. Upp, Fluid Flow Measurement, 2nd ed., Butterworth-Heinemann, 2002, pp. $183-212$. 
[8] B. R. Hanson and L. J. Schwankl, "Error Analysis of Flowmeter Measurements," Journal of Irrigation and Drainage Engineering, vol. 124, no. 5, pp. 248-256, 1998.

[9] J. B. Prettyman, "Comparison of Selected Differential Producing, Ultrasonic, and Magnetic Flow Meters," Logan, 2014.

[10] F. Cascetta, A. Palombo and G. Scalabrini, "Water flow measurement in large bore pipes: An experimental comparison between two different types of isertion flowmeters," ISA Transactions, vol. 42, no. 2, pp. 171-179, 2003.

[11] "Ultrasonic | Definition of Ultrasonic," Merriam-Webster, [Online]. Available: http://www.merriam-webster.com/dictionary/ultrasonic. [Accessed 7 February 2016].

[12] B. Looss, C. Lhuillier and H. Jeanneau, "Numerical simulation of transit-time ultrasonic flowmeters: uncertainties due to flow profile and fluid turbulence," Ultrasonics, vol. 40, no. 9, pp. 1009-1015, 2002.

[13] Z. Cao, S. Song, Z. Peng and L. Xu, "Coil shape optimization of the electromagnetic flowmeter for different flow profiles," Flow Measurement and Instrumentation, vol. 40, p. 256/262, 2014.

[14] "Kármán vortex street," Wikipedia, 1 February 2016. [Online]. Available: https://en.wikipedia.org/wiki/K\%C3\%A1rm\%C3\%A1n_vortex_street. [Accessed 1 February 2016].

[15] B. R. Munson, D. F. Young, T. H. Okiishi and W. W. Huebsch, Fundamentals of Fluid Mechanics, 6th ed., John Wiley \& Sons, Inc., 2009, pp. 348-351,386,399,657. 
[16] L. Theodore, Chemical Engineering: The Essential Reference, McGraw-Hill Education, 2014.

[17] L. Wilson, "Power Ultrasonic Driver," 11 October 2012. [Online]. Available: http://www.imajeenyus.com/electronics/20110514_power_ultrasonic_driver/index.shtml. [Accessed 13 January 2015].

[18] "Texas Instruments Literature," TI, November 2015. [Online]. Available: http://www.ti.com/lit/ug/sniu021a/sniu021a.pdf.

[19] "Ultrasonic EVM / Transducer Selector," Texas Instruments, 2014. [Online]. Available: https://webench.ti.com/webench5/TDC/index.html?origin=pf_panel\&app=flowHead. [Accessed 2016].

[20] "TDS-100H Handheld Ultrasonic Flowmeter," [Online]. Available: http://www.idiytools.com/upload/pro/tds-100h-user-manual.pdf.

[21] "zhuomajd," iDiyTools, [Online]. Available: http://www.idiytools.com/upload/pro/tds100h-user-manual.pdf.

[22] "The Fire Service," National Fire Protection Association, [Online]. Available: http://www.nfpa.org/research/reports-and-statistics/the-fire-service.

[23] P. Spurgeon, Fire Service Hydraulics and Pump Operations, Penn Well Corporation, 2012. 


\section{Appendix A - Driver Circuit}

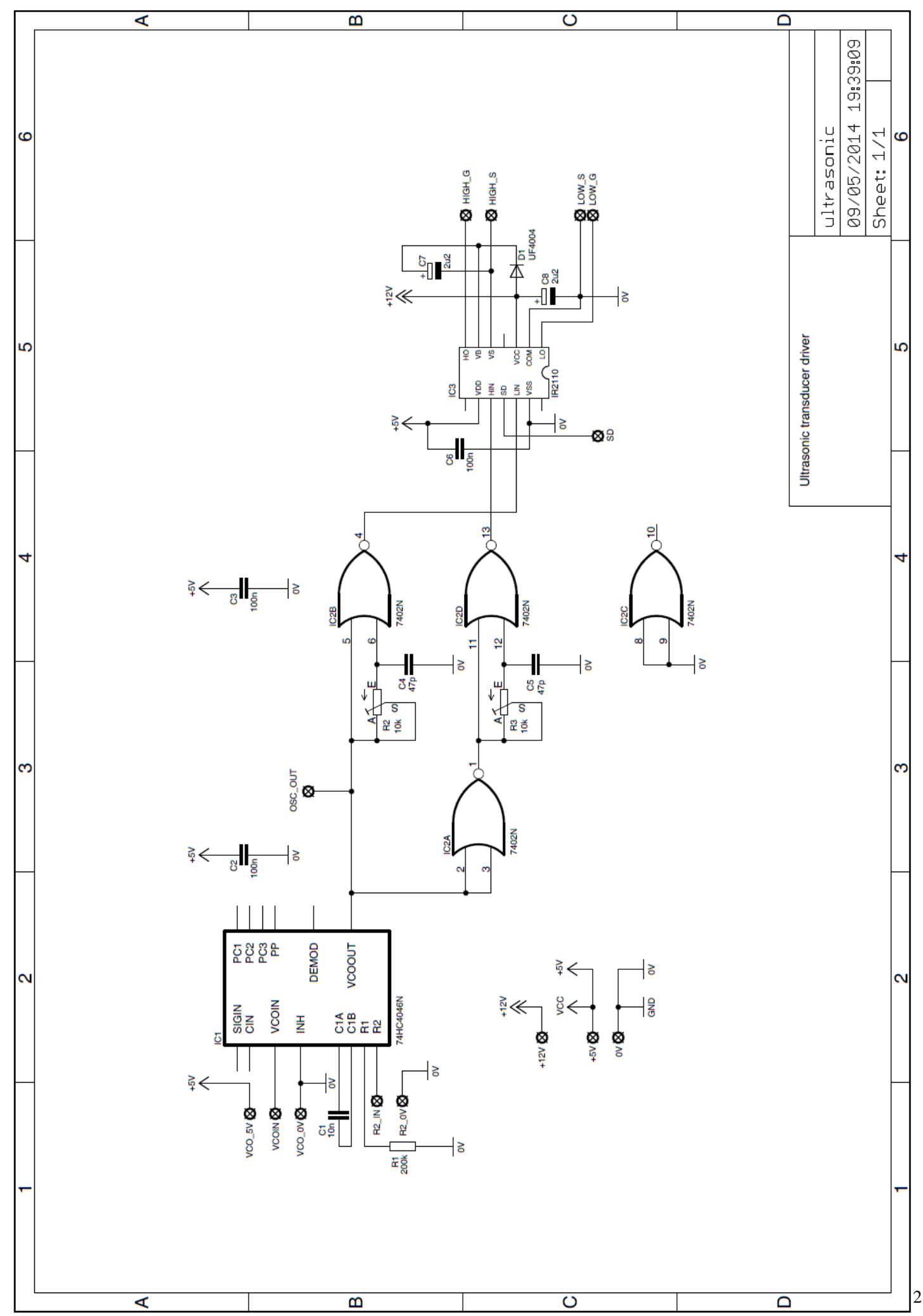

